\title{
Higher-order symmetric duality in nondifferentiable multiobjective fractional programming problem over cone contraints
}

\author{
Ramu Dubey ${ }^{1}$, Deepmala ${ }^{2, *}$, Vishnu Narayan Mishra ${ }^{3}$ \\ ${ }^{1}$ Department of Mathematics, J.C. Bose University of Science and Technology, YMCA, Faridabad, India \\ ${ }^{2}$ Mathematics Discipline, PDPM Indian Institute of Information Technology, Design and Manufacturing, Jabalpur, India \\ ${ }^{3}$ Department of Mathematics, Indira Gandhi National Tribal University, Amarkantak, India
}

\begin{abstract}
In this paper, we introduce the definition of higher-order $K-(C, \alpha, \rho, d)$-convexity/pseudoconvexity over cone and discuss a nontrivial numerical examples for existing such type of functions. The purpose of the paper is to study higher order fractional symmetric duality over arbitrary cones for nondifferentiable Mond-Weir type programs under higherorder $K-(C, \alpha, \rho, d)$-convexity/pseudoconvexity assumptions. Next, we prove appropriate duality relations under aforesaid assumptions.
\end{abstract}

Keywords Higher-order symmetric duality. Multiobjective fractional programming. Efficient solution. Higher-order $K$ $(C, \alpha, \rho, d)$-convexity/pseudoconvexity

AMS 2010 subject classifications 90C26, 90C30, 90C32, 90C46

DOI: $10.19139 /$ soic-2310-5070-601

\section{Introduction}

Convexity and generalized convexity have been playing an important role in developing optimality and duality results for multiobjective programming problems which are mathematical models for most of the real world problems occuring in the fields of engineering, economics, finance, game theory etc. Higher-order duality is significant due to its computational importance as it provides more higher bounds whenever approximation is used. Mangasarian [1] formulated higher-order dual for a single objective nonlinear problems, $\{\min f(x)$, subject to $g(x) \leqq 0\}$. Motivated by this concept, many researchers have worked in this direction. Kassem [3] have been studied higher-order vector optimization problem and derived duality results under generalized convexity assumptions.

In last many years, various optimality and duality results have been obtained for multiobjective fractional programming problems. In Chen [2] multiobjective fractional problem and its duality relations have been considered under higher-order $(f, \alpha, \rho, d)$ - convexity assumptions. Later on, Suneja et al. [4] proved higher-order Mond-Weir and Schaible type nondifferentiable dual programs and their duality relations under higher-order $(f, \rho, \sigma)$-type $I$ - assumptions. Recently, Ying [5] has studied higher-order multiobjective symmetric fractional problem and formulated its Mond- Weir type dual and duality theorems are proved under the higher-order $(f, \alpha, \rho, d)$-convexity assumptions. Several reseachers worked in the same fields[ [11]- [15]].

\footnotetext{
*Correspondence to: Deepmala (Email: dmrai23@gmail.com, deepmala@iiitdmj.ac.in). Mathematics Discipline, PDPM-Indian Institute of Information Technology, Design and Manufacturing, Jabalpur, Dumna Airport Road, P.O.: Khamaria, Jabalpur 482 005, Madhya Pradesh, India.
}

ISSN 2310-5070 (online) ISSN 2311-004X (print)

Copyright (C) 2020 International Academic Press 
In the present work, we formulate a pair of nondifferentiable multiobjective Mond-Weir type higher-order symmetric fractional programming problems over arbitrary cones. For a differentiable function $h: X \times R^{n} \longrightarrow$ $R,\left(X \subset R^{n}\right)$, we introduce the definition of higher-order $K-(C, \alpha, \rho, d)$-convexity/pseudoconvexity, which extends some kinds of generalized convexity. Also, we give nontrivial concrete numerical examples which is higher-order $K-(C, \alpha, \rho, d)$ - convex/pseudoconvex function, but it is neither higher-order $(C, \alpha, \rho, d)$ convex/pseudoconvex function nor higher-order $K-(F, \alpha, \rho, d) /(F, \alpha, \rho, d)$ - convex/pseudoconvex function. Finally, we establish appropriate duality theorems under higher-order $K-(C, \alpha, \rho, d)$ convexity/pseudoconvexity assumptions followed by conclusions.

\section{Preliminaries}

Let $P$ be a pointed convex cone with non empty interior in $R_{+}^{p}$. Then, for $x, y \in R^{p}$, we define three cone orders with respect to $P$ as follows:

$$
\begin{array}{lll}
x<y & \text { if and only if } & y-x \in \operatorname{int} P, \\
x \leq y & \text { if and only if } & y-x \in P \backslash\{0\} \\
x \leqq y & \text { if and only if } & y-x \in P .
\end{array}
$$

Definition 2.1[9]. Let $C$ be a compact convex set in $R^{n}$. The support function of $C$ is defined by

$$
s(x \mid C)=\max \left\{x^{T} y: y \in C\right\} .
$$

A support function, being convex and everywhere finite, has a subdifferential, that is, there exists a $z \in R^{n}$ such that

$$
s(y \mid C) \geqq s(x \mid C)+z^{T}(y-x), \forall x \in C .
$$

The subdifferential of $s(x \mid C)$ is given by

$$
\partial s(x \mid C)=\left\{z \in C: z^{T} x=s(x \mid C)\right\} .
$$

For a convex set $D \subset R^{n}$, the normal cone to $D$ at a point $x \in D$ is defined by

$$
N_{D}(x)=\left\{y \in R^{n}: y^{T}(z-x) \leqq 0, \forall z \in D\right\} .
$$

When $C$ is a compact convex set, $y \in N_{C}(x)$ if and only if $s(y \mid C)=x^{T} y$, or equivalently, $x \in \partial s(y \mid C)$.

Definition 2.2.[9]. The positive polar cone $P^{*}$ of a cone $P$ is defined by

$$
P^{*}=\left\{y \in R^{p}: x^{T} y \geqq 0, \forall x \in P\right\}
$$

Now, consider the following multiobjective programming problem:

$$
\begin{aligned}
& \left(P_{1}\right) \quad K-\text { Minimize } f(x) \\
& \quad \text { subject to } x \in X^{0}=\{x \in S:-g(x) \in M\}
\end{aligned}
$$

where $S \subset R^{n}$ is open, $f: S \rightarrow R^{k}, g: S \rightarrow R^{m}, K$ and $M$ are closed convex pointed cones with nonempty interiors in $R^{k}$ and $R^{m}$, respectively.

Definition 2.3. A feasible solution $\bar{x} \in X^{0}$ is said to be an efficient solution of $\left(P_{1}\right)$ if there exists no $x \in X^{0}$ such that $f(x)-f(\bar{x}) \in K \backslash\{0\}$.

Definition 2.4[10]. Let $C: X \times X \times R^{n} \rightarrow R \quad\left(X \subseteq R^{n}\right)$ be a function which satisfies $C_{x, u}(0)=0$, 
$\forall(x, u) \in X \times X$. Then, the function $C$ is said to be convex on $R^{n}$ with respect to third argument iff for any fixed $(x, u) \in X \times X$,

$$
C_{x, u}\left(\lambda x_{1}+(1-\lambda) x_{2}\right) \leqq \lambda C_{x, u}\left(x_{1}\right)+(1-\lambda) C_{x, u}\left(x_{2}\right), \forall \lambda \in(0,1), \forall x_{1}, x_{2} \in R^{n} .
$$

Many generalizations of the definition of a convex function have been introduced in optimization theory in order to weak the assumption of convexity for establishing optimality and duality results for new classes of nonconvex optimization problems, including vector optimization problems. One of such a generalization of convexity in the vectorial case, we introduce the following concept of higher-order $K-(C, \alpha, \rho, d)$ - convex/pseudoconvex functions:

Definition 2.5. A differentiable function $f: X \rightarrow R^{k}$ is said to be higher order $K-(C, \alpha, \rho, d)$-convex at $u \in X$ with respect to $h: X \times R^{n} \rightarrow R^{k}$ if for all $x \in X$ and $p \in R^{k}, \exists \rho \in R^{k}$, a real valued function $\alpha: X \times X \rightarrow R_{+} \backslash\{0\}$ and $d: X \times X \rightarrow R^{k}$ (satisfying $d(x, z)=0 \Leftrightarrow x=z$ ) such that

$$
\begin{aligned}
& \frac{1}{\alpha(x, u)}\left[f_{1}(x)-f_{1}(u)-h_{1}\left(u, p_{1}\right)+p_{1}^{T} \nabla_{p_{1}} h_{1}\left(u, p_{1}\right)-\rho_{1} d_{1}^{2}(x, u)\right]-C_{x, u}\left[\nabla_{x} f_{1}(u)+\nabla_{p_{1}} h_{1}\left(u, p_{1}\right)\right] \\
& , \ldots, \frac{1}{\alpha(x, u)}\left[f_{k}(x)-f_{k}(u)-h_{k}\left(u, p_{k}\right)+p_{k}^{T} \nabla_{p_{k}} h_{k}\left(u, p_{k}\right)-\rho_{k} d_{k}^{2}(x, u)\right]-C_{x, u}\left[\nabla_{x} f_{k}(u)+\nabla_{p_{k}} h_{k}\left(u, p_{k}\right)\right] \in K .
\end{aligned}
$$

The function $f$ is said to be higher-order $K-(C, \alpha, \rho, d)$ - convex over $X$ if, $\forall u \in X$, it is higher $K-(C, \alpha, \rho, d)-$ convex.

The following example shows that $\exists$ functions which are higher-order $K-(C, \alpha, \rho, d)$ - convex function, but the functions do not others ( such as higher-order $K-(F, \alpha, \rho, d) /(F, \alpha, \rho, d)$ - convex functions and higherorder $(C, \alpha, \rho, d)$ - convex functions).

Example 2.1. Let $X=[0,5]$ and $K=\{(x, y):|y| \leqq 20 x$ and $x \geqq 0\}$.

Consider the function $f=\left(f_{1}, f_{2}\right) \rightarrow R^{2}$ given by

$$
f_{1}(x)=i\left(e^{-i x}-e^{i x}\right), f_{2}(x)=i\left(e^{i x}-e^{-i x}\right) .
$$

Let the convex function $C: X \times X \times R \rightarrow R$ be defined by

$$
C_{x, u}(a)=\frac{a^{2}}{4}(x-u) .
$$

Further, the function $h=\left(h_{1}, h_{2}\right): X \times R^{n} \rightarrow R^{2}$ be defined as

$$
h_{1}\left(u, p_{1}\right)=\frac{u^{2}}{2} p_{1}, h_{2}\left(u, p_{2}\right)=-u^{2} p_{2} .
$$

Next $\alpha(x, u)=2, d_{i}(x, u)=|x-u|, i=1,2$ and $\rho_{i}=0, i=1,2$.

We will prove that the function $f=\left(f_{1}, f_{2}\right)$ is higher-order $K-(C, \alpha, \rho, d)$-convex function at $u=0$. For this, we have to claim that

$$
\begin{aligned}
& \Pi=\left\{\frac{1}{\alpha(x, u)}\left[f_{1}(x)-f_{1}(u)-h_{1}\left(u, p_{1}\right)+p_{1}^{T} \nabla_{p_{1}} h_{1}\left(u, p_{1}\right)-\rho_{1} d_{1}^{2}(x, u)\right]-C_{x, u}\left[\nabla_{x} f_{1}(u)+\nabla_{p_{1}} h_{1}\left(u, p_{1}\right)\right],\right. \\
& \left.\frac{1}{\alpha(x, u)}\left[f_{2}(x)-f_{2}(u)-h_{2}\left(u, p_{2}\right)+p_{2}^{T} \nabla_{p_{2}} h_{2}\left(u, p_{2}\right)-\rho_{2} d_{2}^{2}(x, u)\right]-C_{x, u}\left[\nabla_{x} f_{2}(u)+\nabla_{p_{2}} h_{2}\left(u, p_{2}\right)\right]\right\} \in K
\end{aligned}
$$

or

$$
\Pi=\left(\phi_{1}, \phi_{2}\right) \in K
$$




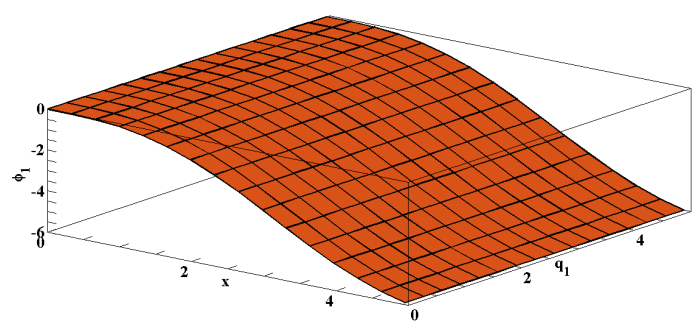

Figure 1. $\phi_{1}=(\sin x-x), \forall x \in[0,5]$

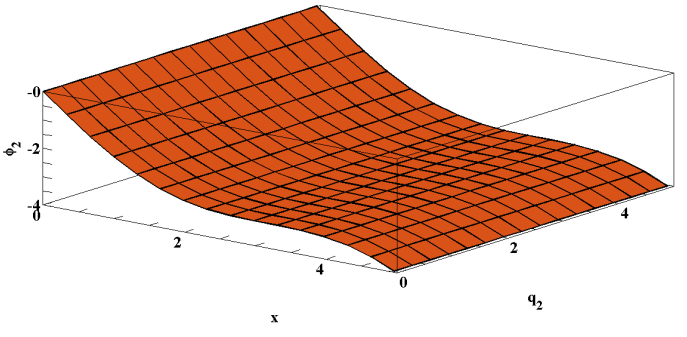

Figure 2. $\phi_{2}=(-\sin x-x), \forall x \in[0,5]$

where

$$
\phi_{1}=\frac{1}{\alpha(x, u)}\left[f_{1}(x)-f_{1}(u)-h_{1}\left(u, p_{1}\right)+p_{1}^{T} \nabla_{p_{1}} h_{1}\left(u, p_{1}\right)-\rho_{1} d_{1}^{2}(x, u)\right]-C_{x, u}\left[\nabla_{x} f_{1}(u)+\nabla_{p_{1}} h_{1}\left(u, p_{1}\right)\right]
$$

and

$$
\phi_{2}=\frac{1}{\alpha(x, u)}\left[f_{2}(x)-f_{2}(u)-h_{2}\left(u, p_{2}\right)+p_{2}^{T} \nabla_{p_{2}} h_{2}\left(u, p_{2}\right)-\rho_{2} d_{2}^{2}(x, u)\right]-C_{x, u}\left[\nabla_{x} f_{2}(u)+\nabla_{p_{2}} h_{2}\left(u, p_{2}\right)\right]
$$

Substituting the values $f_{1}, f_{2}, h_{1}, h_{2}, \alpha, \rho_{1}, \rho_{2}$ and $d_{i}(x, u), i=1,2$ in the above expressions, we have

$$
\phi_{1}=\frac{1}{2}\left[i\left(e^{-i x}-e^{i x}\right)-i\left(e^{-i u}-e^{i u}\right)-\frac{u^{2}}{2} p_{1}+\frac{u^{2}}{2} p_{1}-0 \times(x-u)^{2}\right]-C_{x, u}\left[\left(e^{-i u}+e^{i u}\right)+\frac{u^{2}}{2}\right]
$$

and

$$
\phi_{2}=\frac{1}{2}\left[i\left(e^{i x}-e^{-i x}\right)-i\left(e^{i u}-e^{-i u}\right)-u^{2} p_{2}+u^{2} p_{2}-0 \times\left((x-u)^{2}\right]-C_{x, u}\left[-\left(e^{i u}+e^{i u}\right)+u^{2}\right] .\right.
$$

At the point at $u=0$, we have

$$
\phi_{1}=\frac{1}{2}\left[i\left(e^{-i x}-e^{i x}\right)\right]-C_{x, u}[2]
$$

and

$$
\phi_{2}=\frac{1}{2}\left[-\left(e^{i x}-e^{-i x}\right)\right]-C_{x, u}[-2] .
$$

Using the condition $C_{x, u}(a)=\frac{a^{2}}{4}(x-u)$ in above expressions,

$$
\phi_{1}=(\sin x-x)
$$

and

$$
\phi_{2}=(-\sin x-x) .
$$

Obviously, from the given figures (1) and (2), it follows that

$$
\Pi=\left(\phi_{1}, \phi_{2}\right) \in K, \forall x \in X
$$

or

$$
\Pi=(\sin x-x,-\sin x-x) \in K .
$$

This shows that $f=\left(f_{1}, f_{2}\right)$ is higher-order $K-(C, \alpha, \rho, d)$ - convex function at $u=0$.

Obviously, $\Pi=(\sin x-x,-\sin x-x) \supsetneqq 0, \forall x \in X$. This implies the $f=\left(f_{1}, f_{2}\right)$ is not higher-order 


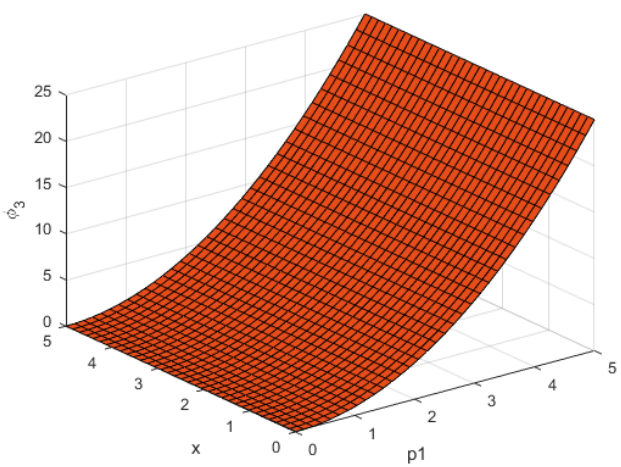

Figure 3. $\phi_{3}=x^{2}, \forall x \in[0,5]$

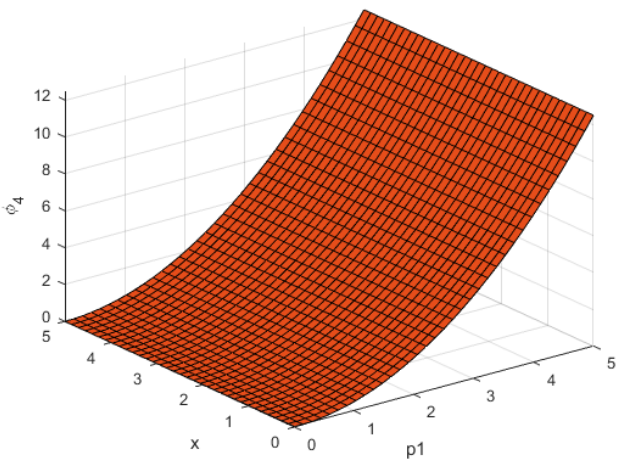

Figure 4. $\phi_{4}=\frac{x^{2}}{4}, \forall x \in[0,5]$

$(C, \alpha, \rho, d)$ - convex function at the point $u=0$. Next, the function $C_{x, u}($.$) is not sublinear in the third positions.$ Hence, the function is neither higher-order $K-(F, \alpha, \rho, d)$ - convex function nor higher-order $(F, \alpha, \rho, d)$ - convex function at $u=0$.

Definition 2.6. A differentiable function $f: X \rightarrow R^{k},\left(X \subseteq R^{n}\right)$ is said to be higher order $K-(C, \alpha, \rho, d)$ pseudoconvex at $u \in X$ with respect to $h: X \times R^{n} \rightarrow R^{k}$ if for all $x \in X$ and $p \in R^{k}, \exists \rho \in R^{k}$, a real valued function $\alpha: X \times X \rightarrow R_{+} \backslash\{0\}$ and $d: X \times X \rightarrow R^{k}$ (satisfying $d(x, z)=0 \Leftrightarrow x=z$ ) such that

$$
\begin{aligned}
& \left\{C_{x, u}\left[\nabla_{x} f_{1}(u)+\nabla_{p_{1}} h_{1}\left(u, p_{1}\right)\right], C_{x, u}\left[\nabla_{x} f_{2}(u)+\nabla_{p_{2}} h_{2}\left(u, p_{2}\right)\right], \ldots, C_{x, u}\left[\nabla_{x} f_{k}(u)+\nabla_{p_{k}} h_{k}\left(u, p_{k}\right)\right]\right\} \in K \\
& \Rightarrow\left\{\frac{1}{\alpha(x, u)}\left[f_{1}(x)-f_{1}(u)-h_{1}\left(u, p_{1}\right)+p_{1}^{T} \nabla_{p_{1}} h_{1}\left(u, p_{1}\right)-\rho_{1} d_{1}^{2}(x, u)\right], \frac{1}{\alpha(x, u)}\left[f_{2}(x)-f_{2}(u)-h_{2}\left(u, p_{2}\right)+\right.\right. \\
& \left.\left.p_{2}^{T} \nabla_{p_{2}} h_{2}\left(u, p_{2}\right)-\rho_{2} d_{2}^{2}(x, u)\right], \ldots, \frac{1}{\alpha(x, u)}\left[f_{k}(x)-f_{k}(u)-h_{k}\left(u, p_{k}\right)+p_{k}^{T} \nabla_{p_{k}} h_{k}\left(u, p_{k}\right)-\rho_{k} d_{k}^{2}(x, u)\right]\right\} \in K .
\end{aligned}
$$

Example 2.2. Let $X=[0,5]$ and $K=\{(x, y):|y| \leqq 20 x$ and $x \geqq 0\}$.

Consider the function $f=\left(f_{1}, f_{2}\right) \rightarrow R^{2}$ given by

$$
f_{1}(x)=\left(4+e^{x}-e^{x}\right), f_{2}(x)=\left(\frac{e^{-x}-e^{x}}{2}\right) .
$$

Let the convex function $C: X \times X \times R \rightarrow R$ be defined by

$$
C_{x, u}(a)=\frac{a^{2}}{4}\left(x^{2}+u^{2}\right) .
$$

The function $h=\left(h_{1}, h_{2}\right): X \times R^{n} \rightarrow R^{2}$ is defined as:

$$
h_{1}\left(u, p_{1}\right)=\frac{-u^{2}}{4} p_{1}, h_{2}\left(u, p_{2}\right)=u^{4} p_{2} .
$$

Next, $\alpha(x, u)=2, d_{i}(x, u)=|x+u|, i=1,2$ and $\rho_{i}=0, i=1,2$.

In order to prove that the function $f=\left(f_{1}, f_{2}\right)$ is higher-order $K-(C, \alpha, \rho, d)$-pseudo convex function at 


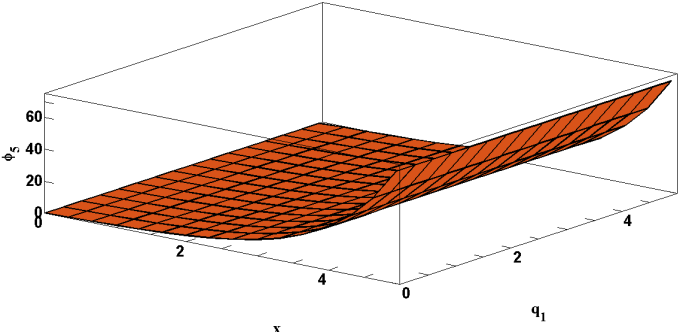

Figure 5. $\phi_{5}=\sinh x, \forall x \in[0,5]$

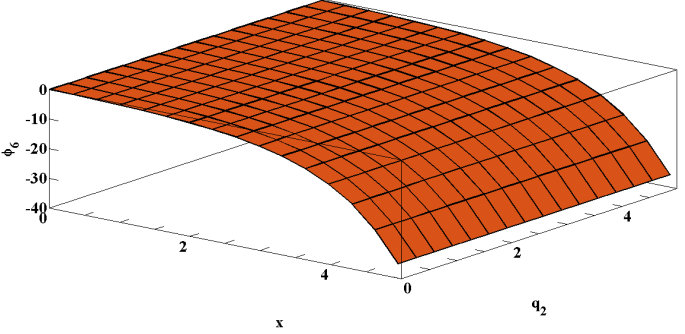

Figure 6. $\phi_{6}=\frac{-\sinh x}{2}, \forall x \in[0,5]$

$u=0$. For this, we have to show that

$$
\begin{aligned}
\Upsilon=\{ & \left.C_{x, u}\left[\nabla_{x} f_{1}(u)+\nabla_{p_{1}} h_{1}\left(u, p_{1}\right)\right], C_{x, u}\left[\nabla_{x} f_{2}(u)+\nabla_{p_{2}} h_{2}\left(u, p_{2}\right)\right]\right\} \in K \\
\Rightarrow \Gamma= & \left\{\frac{1}{\alpha(x, u)}\left[f_{1}(x)-f_{1}(u)-h_{1}\left(u, p_{1}\right)+p_{1}^{T} \nabla_{p_{1}} h_{1}\left(u, p_{1}\right)-\rho_{1} d_{1}^{2}(x, u)\right], \frac{1}{\alpha(x, u)}\left[f_{2}(x)-f_{2}(u)\right.\right. \\
& \left.\left.-h_{2}\left(u, p_{2}\right)+p_{2}^{T} \nabla_{p_{2}} h_{2}\left(u, p_{2}\right)-\rho_{2} d_{2}^{2}(x, u)\right]\right\} \in K
\end{aligned}
$$

or

$$
\Upsilon=\left(\phi_{3}, \phi_{4}\right) \in K \Rightarrow \Gamma=\left(\phi_{5}, \phi_{6}\right) \in K
$$

where

$$
\begin{gathered}
\phi_{3}=C_{x, u}\left[\nabla_{x} f_{1}(u)+\nabla_{p_{1}} h_{1}\left(u, p_{1}\right)\right], \phi_{4}=C_{x, u}\left[\nabla_{x} f_{2}(u)+\nabla_{p_{2}} h_{2}\left(u, p_{2}\right)\right], \\
\phi_{5}=\frac{1}{\alpha(x, u)}\left[f_{1}(x)-f_{1}(u)-h_{1}\left(u, p_{1}\right)+p_{1}^{T} \nabla_{p_{1}} h_{1}\left(u, p_{1}\right)-\rho_{1} d_{1}^{2}(x, u)\right]
\end{gathered}
$$

and

$$
\phi_{6}=\frac{1}{\alpha(x, u)}\left[f_{2}(x)-f_{2}(u)-h_{2}\left(u, p_{2}\right)+p_{2}^{T} \nabla_{p_{2}} h_{2}\left(u, p_{2}\right)-\rho_{2} d_{2}^{2}(x, u)\right] .
$$

Substituting the values $f_{1}, f_{2}, h_{1}, h_{2}, \alpha, \rho_{1}, \rho_{2}$ and $d_{i}(x, u), i=1,2$ in the above expressions, we have

$$
\begin{gathered}
\phi_{3}=C_{x, u}\left[\left(e^{u}+e^{u}\right)-\frac{u^{2}}{2}\right], \phi_{4}=C_{x, u}\left[\frac{e^{u}+e^{u}}{2}+u^{4}\right], \\
\phi_{5}=\frac{1}{2}\left[\left(4+e^{x}-e^{x}\right)-\left(4+e^{u}-e^{u}\right)+\frac{u^{2}}{4} p_{1}-\frac{u^{2}}{4} p_{1}-0 \times(x+u)^{2}\right]
\end{gathered}
$$

and

$$
\phi_{6}=\frac{1}{2}\left[\frac{e^{-x}-e^{x}}{2}-\left(\frac{e^{-u}-e^{u}}{2}\right)-u^{4} p_{2}+u^{4} p_{2}-0 \times(x+u)^{2}\right]
$$

which at $u=0$, yields

$$
\phi_{3}=C_{x, u}(2), \quad \phi_{4}=C_{x, u}(1), \quad \phi_{5}=\left(\frac{e^{x}-e^{x}}{2}\right)
$$

and

$$
\phi_{6}=\left(\frac{e^{-x}-e^{x}}{4}\right)
$$


Using the condition $C_{x, u}(a)=\frac{a^{2}}{4}\left(x^{2}+u^{2}\right)$ in above expressions,

$$
\phi_{3}=x^{2}, \phi_{4}=\left(\frac{x^{2}}{4}\right), \phi_{5}=\sinh x
$$

and

$$
\phi_{6}=\left(\frac{-\sinh x}{2}\right)
$$

Further,

$$
\Upsilon=\left(x^{2}, \frac{x^{2}}{4}\right) \in K \quad(\text { from figures }(3) \text { and }(4))
$$

and

$$
\Gamma=\left(\sinh x, \frac{-\sinh x}{2}\right) \in K(\text { from figures }(5) \text { and }(6)) .
$$

This gives that

$$
\Upsilon=\left(x^{2}, \frac{x^{2}}{4}\right) \in K \Rightarrow \Gamma=\left(\sinh x, \frac{-\sinh x}{2}\right) \in K
$$

or

$$
\Upsilon=\left(\phi_{3}, \phi_{4}\right) \in K \Rightarrow \Gamma=\left(\phi_{5}, \phi_{6}\right) \in K .
$$

Therefore, $f=\left(f_{1}, f_{2}\right)$ is higher-order $K-(C, \alpha, \rho, d)$ - pseudoconvex function at $u=0$.

Next, $\Upsilon=\left(\sinh x, \frac{-\sinh x}{2}\right) \supsetneqq 0, \forall x \in X$ from the figures. This shows that the function $f=\left(f_{1}, f_{2}\right)$ is not higher-order $(C, \alpha, \rho, d)$ - pseudoconvex function at the point $u=0$. Furthermore, the function $C_{x, u}($.$) is$ not sublinear with respect to third variables. Therefore, the function is neither higher-order $K-(F, \alpha, \rho, d)$ pseudoconvex function nor higher-order $(F, \alpha, \rho, d)$ - pseudoconvex function at $u=0$.

\section{Remark 2.1.}

(i) If $K=R^{+}$, then the Definition 2.5 in reduces in higher-order $(C, \alpha, \rho, d)$-convexity given by [6].

(ii) If $C_{x, u}(a)=\eta(x, u) T a, h_{i}\left(u, p_{i}\right)=\frac{1}{2} p_{i}^{T} \nabla f_{i}(u) p_{i}, k=1,2, \ldots, k, \rho=0$ and $\alpha(x, u)=1$ then Definition 2.5 becomes $K-\eta$ bonvexity given by [8].

\section{Higher-order Mond-Weir fractional symmetric duality}

Consider the following multiobjective fractional symmetric dual programs over arbitrary cones:

(MFPP) K-minimize $R(x, y, p)=\left(R_{1}\left(x, y, p_{1}\right), R_{2}\left(x, y, p_{2}\right), \ldots, R_{k}\left(x, y, p_{k}\right)\right)^{T}$ subject to

$$
\begin{gathered}
-\sum_{i=1}^{k} \lambda_{i}\left[\left(\nabla_{y} f_{i}(x, y)-z_{i}+\nabla_{p_{i}} H_{i}\left(x, y, p_{i}\right)\right)-R_{i}\left(x, y, p_{i}\right)\left(\nabla_{y} g_{i}(x, y)+r_{i}+\nabla_{p_{i}} G_{i}\left(x, y, p_{i}\right)\right)\right] \in C_{2}^{*}, \\
y^{T}\left[\sum_{i=1}^{k} \lambda_{i}\left[\left(\nabla_{y} f_{i}(x, y)-z_{i}+\nabla_{p_{i}} H_{i}\left(x, y, p_{i}\right)\right)-R_{i}\left(x, y, p_{i}\right)\left(\nabla_{y} g_{i}(x, y)+r_{i}+\nabla_{p_{i}} G_{i}\left(x, y, p_{i}\right)\right)\right]\right] \geqq 0, \\
\lambda \in \operatorname{int} K^{*}, x \in C_{1}, z_{i} \in D_{i}, r_{i} \in F_{i}, i=1,2, \ldots, k
\end{gathered}
$$


. (MFDP) $K$-maximize $S(u, v, q)=\left(S_{1}\left(u, v, q_{1}\right), S_{2}\left(u, v, q_{2}\right), \ldots, S_{k}\left(u, v, q_{k}\right)\right)^{T}$ subject to

$$
\begin{gathered}
\sum_{i=1}^{k} \lambda_{i}\left[\left(\nabla_{x} f_{i}(u, v)+w_{i}+\nabla_{q_{i}} \Phi_{i}\left(u, v, q_{i}\right)\right)-S_{i}\left(u, v, q_{i}\right)\left(\nabla_{x} g_{i}(u, v)-t_{i}+\nabla_{q_{i}} \Psi_{i}\left(u, v, q_{i}\right)\right)\right] \in C_{1}^{*}, \\
u^{T}\left[\sum_{i=1}^{k} \lambda_{i}\left[\left(\nabla_{x} f_{i}(u, v)+w_{i}+\nabla_{q_{i}} \Phi_{i}\left(u, v, q_{i}\right)\right)-S_{i}\left(u, v, q_{i}\right)\left(\nabla_{x} g_{i}(u, v)-t_{i}+\nabla_{q_{i}} \Psi_{i}\left(u, v, q_{i}\right)\right)\right]\right] \leqq 0, \\
\lambda \in \operatorname{int} K^{*}, v \in C_{2}, w_{i} \in Q_{i}, t_{i} \in E_{i}, i=1,2, \ldots, k,
\end{gathered}
$$

where

$$
\begin{aligned}
R_{i}\left(x, y, p_{i}\right) & =\frac{f_{i}(x, y)+s\left(x \mid Q_{i}\right)-y^{T} z_{i}+H_{i}\left(x, y, p_{i}\right)-p_{i}^{T} \nabla_{p_{i}} H_{i}\left(x, y, p_{i}\right)}{g_{i}(x, y)-s\left(x \mid E_{i}\right)+y^{T} r_{i}+G_{i}\left(x, y, p_{i}\right)-p_{i}^{T} \nabla_{p_{i}} G_{i}\left(x, y, p_{i}\right)}, \\
S_{i}\left(u, v, q_{i}\right) & =\frac{f_{i}(u, v)-s\left(v \mid D_{i}\right)+u^{T} w_{i}+\Phi_{i}\left(u, v, q_{i}\right)-q_{i}^{T} \nabla_{q_{i}} \Phi_{i}\left(u, v, q_{i}\right)}{g_{i}(u, v)+s\left(v \mid F_{i}\right)-u^{T} t_{i}+\Psi_{i}\left(u, v, q_{i}\right)-q_{i}^{T} \nabla_{q_{i}} \Psi_{i}\left(u, v, q_{i}\right)},
\end{aligned}
$$

where $f_{i}: S_{1} \times S_{2} \rightarrow R ; g_{i}: S_{1} \times S_{2} \rightarrow R ; H_{i}, G_{i}: S_{1} \times S_{2} \times R^{m} \rightarrow R$ and $\Phi_{i}, \Psi_{i}: S_{1} \times S_{2} \times R^{n} \rightarrow R$ are differentiable functions for all $i=1,2, \ldots, k . S_{1} \subseteq R^{n}$ and $S_{2} \subseteq R^{m}$ are such that $C_{1} \times C_{2} \subset S_{1} \times S_{2} . Q_{i}, E_{i}$ are compact convex sets in $R^{n}$ and $D_{i}, F_{i}$ are compact convex sets in $R^{m}, p_{i} \in R^{n}, q_{i} \in R^{m}, i=1,2, \ldots, k$, $p=\left(p_{1}, p_{2}, \ldots, p_{k}\right), q=\left(q_{1}, q_{2}, \ldots, q_{k}\right) . C_{1}^{*}$ and $C_{2}^{*}$ are positive polar cones of $C_{1}$ and $C_{2}$, respectively. It is assumed that in the feasible regions, the numerators are nonnegative and denominators are positive and $K$ is a closed convex cone with $R_{+}^{k} \subseteq K$.

Let $T=\left(T_{1}, T_{2}, \ldots, T_{k}\right)^{T}$ and $W=\left(W_{1}, W_{2}, \ldots, W_{k}\right)^{T}$. Then, we can express the programs (MFPP) and (MFDP) equivalently as:

$(\mathbf{M F P P})_{T} K$ - minimize $T$ subject to

$$
\begin{gathered}
\left.\left(f_{i}(x, y)+s\left(x \mid Q_{i}\right)-y^{T} z_{i}+H_{i}\left(x, y, p_{i}\right)\right)-p_{i}^{T} \nabla_{p_{i}} H_{i}\left(x, y, p_{i}\right)\right) \\
-T_{i}\left(g_{i}(x, y)-s\left(x \mid E_{i}\right)+y^{T} r_{i}+G_{i}\left(x, y, p_{i}\right)-p_{i}^{T} \nabla_{p_{i}} G_{i}\left(x, y, p_{i}\right)\right)=0, i=1,2, \ldots, k, \\
-\sum_{i=1}^{k} \lambda_{i}\left[\nabla_{y} f_{i}(x, y)-z_{i}+\nabla_{p_{i}} H_{i}\left(x, y, p_{i}\right)-T_{i}\left(\nabla_{y} g_{i}(x, y)+r_{i}+\nabla_{p_{i}} G_{i}\left(x, y, p_{i}\right)\right)\right] \in C_{2}^{*}, \\
y^{T}\left[\sum_{i=1}^{k} \lambda_{i}\left[\nabla_{y} f_{i}(x, y)-z_{i}+\nabla_{p_{i}} H_{i}\left(x, y, p_{i}\right)-T_{i}\left(\nabla_{y} g_{i}(x, y)+r_{i}+\nabla_{p_{i}} G_{i}\left(x, y, p_{i}\right)\right)\right]\right] \geqq 0, \\
\lambda \in \operatorname{int} K^{*}, x \in C_{1}, z_{i} \in D_{i}, r_{i} \in F_{i}, i=1,2, \ldots, k .
\end{gathered}
$$

$(\text { MFDP })_{W} K$ - maximize $W$ subject to

$$
\begin{aligned}
& \left(f_{i}(u, v)-s\left(v \mid D_{i}\right)+u^{T} w_{i}+\Phi_{i}\left(u, v, q_{i}\right)-q_{i}^{T} \nabla_{q_{i}} \Phi_{i}\left(u, v, q_{i}\right)\right) \\
& \quad-W_{i}\left(g_{i}(u, v)+s\left(v \mid F_{i}\right)-u^{T} t_{i}+\Psi_{i}\left(u, v, q_{i}\right)-q_{i}^{T} \nabla_{q_{i}} \Psi_{i}\left(u, v, q_{i}\right)\right)=0, i=1,2, \ldots, k,
\end{aligned}
$$




$$
\begin{gathered}
\sum_{i=1}^{k} \lambda_{i}\left[\nabla_{x} f_{i}(u, v)+w_{i}+\nabla_{q_{i}} \Phi_{i}\left(u, v, q_{i}\right)-W_{i}\left(\nabla_{x} g_{i}(u, v)-t_{i}+\nabla_{q_{i}} \Psi_{i}\left(u, v, q_{i}\right)\right)\right] \in C_{1}^{*}, \\
u^{T}\left[\sum_{i=1}^{k} \lambda_{i}\left[\left(\nabla_{x} f_{i}(u, v)+w_{i}+\nabla_{q_{i}} \Phi_{i}\left(u, v, q_{i}\right)\right)-W_{i}\left(\nabla_{x} g_{i}(u, v)-t_{i}+\nabla_{q_{i}} \Psi_{i}\left(u, v, q_{i}\right)\right)\right]\right] \leqq 0, \\
\lambda \in \operatorname{int} K^{*}, v \in C_{2}, w_{i} \in Q_{i}, t_{i} \in E_{i}, i=1,2, \ldots, k .
\end{gathered}
$$

Next, we prove weak, strong and converse duality theorems for $(\mathrm{MFPP})_{T}$ and (MFDP $)_{W}$, which one equally apply to (MFPP) and (MFDP).

Let $z=\left(z_{1}, z_{2}, \ldots, z_{k}\right), \quad r=\left(r_{1}, r_{2}, \ldots, r_{k}\right), \quad w=\left(w_{1}, w_{2}, \ldots, w_{k}\right)$ and $t=\left(t_{1}, t_{2}, \ldots, t_{k}\right) . \quad f: S_{1} \times S_{2} \rightarrow R^{k}$; $g: S_{1} \times S_{2} \rightarrow R^{m} ; H, G: S_{1} \times S_{2} \times R^{m} \rightarrow R^{k}$ and $\Phi, \Psi: S_{1} \times S_{2} \times R^{n} \rightarrow R^{k}$ are differentiable functions.

Theorem 3.1 (Weak duality). Let $(x, y, T, z, r, \lambda, p)$ be feasible for (MFPP) $)_{T}$ and let $(u, v, W, w, t, \lambda, q)$ be feasible for $(\mathrm{MFDP})_{W}$. Let $f(., v)+(.)^{T} w$ be higher order $K-(C, \alpha, \rho, d)-$ convex at $u$ with respect to $\Phi(u, v, q),-W\left(g(., v)-(.)^{T} t\right)$ be higher-order $K-(C, \alpha, \rho, d)$ - convex at $u$ with respect to $-W \Psi(u, v, q),-\left(f(x,)-.(.)^{T} z\right)$ be higher -order $K-(\bar{C}, \bar{\alpha}, \bar{\rho}, \bar{d})$-convex at $y$ with respect to $-H(x, y, p)$ and $T\left(g(x,)+.(.)^{T} r\right)$ be higher -order $K-(\bar{C}, \bar{\alpha}, \bar{\rho}, \bar{d})$ - convex at $y$ with respect to $T G(x, y, p)$ where $C: R^{n} \times R^{n} \times R^{n} \rightarrow R$ and $\bar{C}: R^{m} \times R^{m} \times R^{m} \rightarrow R$. If the following conditions hold:

$$
\begin{gathered}
\text { either } \sum_{i=1}^{k} \lambda_{i}\left[\rho_{i} d_{i}^{2}(x, u)+\bar{\rho}_{i} \bar{d}_{i}^{2}(v, y)\right] \geqq 0 \quad \text { or } \rho_{i} \geqq 0 \text { and } \quad \bar{\rho}_{i} \geqq 0, i=1,2, \ldots, k, \\
C_{x, u}(a)+a^{T} u \geqq 0, \forall a \in C_{1}^{*}, \bar{C}_{v, y}(b)+b^{T} y \geqq 0, \forall b \in C_{2}^{*} .
\end{gathered}
$$

Then, $T-W \notin-K \backslash\{0\}$.

\section{Proof}

Since $f(., v)+(.)^{T} w$ and $-W\left(g(., v)-(.)^{T} t\right)$ is higher-order $K-(C, \alpha, \rho, d)-$ convex in the first variable at $u$ for fixed $v$, we have

$$
\begin{gathered}
\frac{1}{\alpha(x, u)}\left[f_{1}(x, v)+x^{T} w_{1}-f_{1}(u, v)-u^{T} w_{1}-\Phi_{1}\left(u, v, q_{1}\right)+q_{1}^{T} \nabla_{q_{1}} \Phi_{1}\left(u, v, q_{1}\right)-\rho_{1} d_{1}^{2}(x, u)\right] \\
-C_{x, u}\left(\nabla_{x} f_{1}(u, v)+w_{1}+\nabla_{q_{1}} \Phi_{1}\left(u, v, q_{1}\right)\right), \ldots, \frac{1}{\alpha(x, u)}\left[f_{k}(x, v)+x^{T} w_{k}-f_{k}(u, v)-u^{T} w_{k}-\Phi_{k}\left(u, v, q_{k}\right)\right. \\
\left.\quad+q_{k}^{T} \nabla_{q_{k}} \Phi_{k}\left(u, v, q_{k}\right)-\rho_{k} d_{k}^{2}(x, u)\right]-C_{x, u}\left(\nabla_{x} f_{k}(u, v)+w_{k}+\nabla_{q_{k}} \Phi_{k}\left(u, v, q_{k}\right)\right) \in K .
\end{gathered}
$$

and

$$
\begin{aligned}
& \frac{1}{\alpha(x, u)}\left[W_{1}\left(-g_{1}(x, v)+x^{T} t_{1}+g_{1}(u, v)-u^{T} t_{1}\right)+W_{1}\left(\Psi_{1}\left(u, v, q_{1}\right)-q_{1}^{T} \nabla_{q_{1}} \Psi_{1}\left(u, v, q_{1}\right)\right)-\rho_{1} d_{1}^{2}(x, u)\right] \\
& -C_{x, u}\left(W_{1}\left(-\nabla_{x} g_{1}(u, v)+t_{1}\right)-W_{1} \nabla_{q_{1}} \Psi_{1}\left(u, v, q_{1}\right)\right), \ldots, \frac{1}{\alpha(x, u)}\left[W_{k}\left(-g_{k}(x, v)+x^{T} t_{k}+g_{k}(u, v)-u^{T} t_{k}\right)\right. \\
& \left.+W_{k}\left(\Psi_{k}\left(u, v, q_{k}\right)-q_{k}^{T} \nabla_{q_{k}} \Psi_{k}\left(u, v, q_{k}\right)\right)-\rho_{k} d_{k}^{2}(x, u)\right]-C_{x, u}\left(W_{k}\left(-\nabla_{x} g_{k}(u, v)+t_{k}\right)-W_{k} \nabla_{q_{k}} \Psi_{k}\left(u, v, q_{k}\right)\right) \in K
\end{aligned}
$$


Since $\lambda \in \operatorname{int} K^{*}$, therefore (9) and (10) yield

$$
\begin{aligned}
\sum_{i=1}^{k} \frac{\lambda_{i}}{\alpha(x, u)}\left(f_{i}(x, v)\right. & \left.+x^{T} w_{i}-f_{i}(u, v)-u^{T} w_{i}-\Phi_{i}\left(u, v, q_{i}\right)+q_{i}^{T} \nabla_{q_{i}} \Phi_{i}\left(u, v, q_{i}\right)\right) \\
& -\sum_{i=1}^{k} \frac{\lambda_{i}}{\alpha(x, u)} \rho_{i} d_{i}^{2}(x, u) \geqq \sum_{i=1}^{k} \lambda_{i} C_{x, u}\left(\nabla_{x} f_{i}(u, v)+w_{i}+\nabla_{q_{i}} \Phi_{i}\left(u, v, q_{i}\right)\right) .
\end{aligned}
$$

and

$$
\begin{aligned}
\sum_{i=1}^{k} \frac{\lambda_{i} W_{i}}{\alpha(x, u)}[- & \left.g_{i}(x, v)+x^{T} t_{i}+g_{i}(u, v)-u^{T} t_{i}+\Psi_{i}\left(u, v, q_{i}\right)-q_{i}^{T} \nabla_{q_{i}} \Psi_{i}\left(u, v, q_{i}\right)\right] \\
& -\sum_{i=1}^{k} \frac{\lambda_{i}}{\alpha(x, u)} \rho_{i} d_{i}^{2}(x, u) \geqq \sum_{i=1}^{k} \lambda_{i} C_{x, u}\left(W_{i}\left(-\nabla_{x} g_{i}(u, v)+t_{i}-\nabla_{q_{i}} \Psi_{i}\left(u, v, q_{i}\right)\right)\right) .
\end{aligned}
$$

Now, adding the above two inequalities and then multiplying with $\frac{1}{\tau}$, where $\tau=\sum_{i=1}^{k} \lambda_{i}>0$ as $\lambda \in \operatorname{int} K^{*} \subseteq \operatorname{int} R_{+}^{k}$ and using convexity of $C$, we obtain

$$
\begin{aligned}
& \sum_{i=1}^{k} \frac{\lambda_{i}}{\alpha(x, u) \tau}\left[f_{i}(x, v)+x^{T} w_{i}-f_{i}(u, v)-u^{T} w_{i}-\Phi_{i}\left(u, v, q_{i}\right)+q_{i}^{T} \nabla_{q_{i}} \Phi_{i}\left(u, v, q_{i}\right)\right] \\
& +\sum_{i=1}^{k} \frac{\lambda_{i} W_{i}}{\alpha(x, u) \tau}\left[-g_{i}(x, v)+x^{T} t_{i}+g_{i}(u, v)-u^{T} t_{i}+\Psi_{i}\left(u, v, q_{i}\right)-q_{i}^{T} \nabla_{q_{i}} \Psi_{i}\left(u, v, q_{i}\right)\right]-2 \sum_{i=1}^{k} \frac{\lambda_{i}}{\alpha(x, u) \tau} \rho_{i} d_{i}^{2}(x, u) \\
& \quad \geqq C_{x, u}\left[\sum_{i=1}^{k} \frac{\lambda_{i}}{\tau}\left(\left(\nabla_{x} f_{i}(u, v)+w_{i}+\nabla_{q_{i}} \Phi_{i}\left(u, v, q_{i}\right)\right)-W_{i}\left(\nabla_{x} g_{i}(u, v)-t_{i}+\nabla_{q_{i}} \Psi_{i}\left(u, v, q_{i}\right)\right)\right)\right]
\end{aligned}
$$

Now, from (8) as $\tau>0$, we have

$$
a=\sum_{i=1}^{k} \frac{\lambda_{i}}{\tau}\left[\left(\nabla_{x} f_{i}(u, v)+w_{i}+\nabla_{q_{i}} \Phi_{i}\left(u, v, q_{i}\right)-W_{i}\left(\nabla_{x} g_{i}(u, v)-t_{i}+\nabla_{q_{i}} \Psi_{i}\left(u, v, q_{i}\right)\right)\right] \in C_{1}^{*} .\right.
$$

Hence, for this $a, C_{x, u}(a) \geqq-u^{T} a \geqq 0$ (from (6)). Using this, in (11), we obtain

$$
\begin{aligned}
& \sum_{i=1}^{k} \frac{\lambda_{i}}{\alpha(x, u) \tau}\left(f_{i}(x, v)+x^{T} w_{i}-f_{i}(u, v)-u^{T} w_{i}-\Phi_{i}\left(u, v, q_{i}\right)+q_{i}^{T} \nabla_{q_{i}} \Phi_{i}\left(u, v, q_{i}\right)\right) \\
& +\sum_{i=1}^{k} \frac{\lambda_{i} W_{i}}{\alpha(x, u) \tau}\left[-g_{i}(x, v)+x^{T} t_{i}+g_{i}(u, v)\right. \\
& \left.\quad-u^{T} t_{i}+\Psi_{i}\left(u, v, q_{i}\right)-q_{i}^{T} \nabla_{q_{i}} \Psi_{i}\left(u, v, q_{i}\right)\right] \geqq 2 \sum_{i=1}^{k} \frac{\lambda_{i}}{\alpha(x, u) \tau} \rho_{i} d_{i}^{2}(x, u) .
\end{aligned}
$$

Since $v^{T} r_{i} \leqq s\left(v \mid F_{i}\right)$ and using (4) in above inequality, we get

$$
\sum_{i=1}^{k} \lambda_{i}\left[f_{i}(x, v)+x^{T} w_{i}-s\left(v \mid D_{i}\right)+W_{i}\left(x^{T} t_{i}-v^{T} r_{i}-g_{i}(x, v)\right)\right] \geqq 2 \sum_{i=1}^{k} \lambda_{i} \rho_{i} d_{i}^{2}(x, u) .
$$


Similarly, by the higher-order $K-(\bar{C}, \bar{\alpha}, \bar{\rho}, \bar{d})$ - convexity of $-f(x,)+.(.)^{T} z$ and $T\left(g(x,)+.(.)^{T} r\right)$ in the second variable at $y$, for fixed $x$ and from the condition (8), for

$$
b=-\sum_{i=1}^{k} \frac{\lambda_{i}}{\tau}\left[\left(\nabla_{y} f_{i}(x, y)-z_{i}+\nabla_{p_{i}} H_{i}\left(x, y, p_{i}\right)-T_{i}\left(\nabla_{y} g_{i}(x, y)+r_{i}+\nabla_{p_{i}} G_{i}\left(x, y, p_{i}\right)\right)\right] \in C_{2}^{*},\right.
$$

we get

$$
\sum_{i=1}^{k} \lambda_{i}\left[-f_{i}(x, v)+v^{T} z_{i}-s\left(x \mid Q_{i}\right)+T_{i}\left(v^{T} r_{i}-x^{T} t_{i}+g_{i}(x, v)\right)\right] \geqq 2 \sum_{i=1}^{k} \lambda_{i} \bar{\rho}_{i} \bar{d}_{i}^{2}(v, y) .
$$

Adding the inequalities (12)-(13) and applying (7), we get

$$
\sum_{i=1}^{k} \lambda_{i}\left(v^{T} z_{i}-s\left(v \mid D_{i}\right)+x^{T} w_{i}-s\left(x \mid Q_{i}\right)\right)+\sum_{i=1}^{k} \lambda_{i}\left(T_{i}-W_{i}\right)\left(g_{i}(x, v)+v^{T} r_{i}-x^{T} t_{i}\right) \geqq 0 .
$$

Since $\lambda>0$ and $v^{T} z_{i} \leqq s\left(v \mid D_{i}\right), x^{T} w_{i} \leqq s\left(x \mid Q_{i}\right)$, the above inequality gives

$$
\sum_{i=1}^{k} \lambda_{i}\left(T_{i}-W_{i}\right)\left(g_{i}(x, v)+v^{T} r_{i}-x^{T} t_{i}\right) \geqq 0 .
$$

Using $\left(g_{i}(x, v)+v^{T} r_{i}-x^{T} t_{i}\right)>0, i=1,2, \ldots, k$, above inequality gives

$$
\sum_{i=1}^{k} \lambda_{i}\left(T_{i}-W_{i}\right) \geqq 0
$$

Now, suppose on contrary

$$
T-W \in-K \backslash\{0\}
$$

Since $\lambda>0$, we have

$$
\sum_{i=1}^{k} \lambda_{i}\left(T_{i}-W_{i}\right)<0 .
$$

which contradicts (15). This completes the proof.

Theorem 3.2 (Weak duality). Let $(x, y, T, z, r, \lambda, p)$ and $(u, v, W, w, t, \lambda, q)$ be feasible solutions of (MFPP) $)_{T}$ and $(\mathrm{MFDP})_{W}$, respectively. Suppose that

(i) $\left(f(., v)+(.)^{T} w\right)-W\left(g(., v)-(.)^{T} t\right)$ is higher-order $K-(C, \alpha, \rho, d)$ - convex at $u$ with respect to $(\Phi(u, v, q)-W \Psi(u, v, q))$

(ii) $\left(-f(x,)+.(.)^{T} z\right)+T\left(g(x,)+.(.)^{T} r\right)$ is higher-order $K-(\bar{C}, \bar{\alpha}, \bar{\rho}, \bar{d})$-convex at $y$ with respect to $-H(x, y, p)+T G(x, y, p)$,

(iii) either $\sum_{i=1}^{k} \lambda_{i}\left[\rho_{i} d_{i}^{2}(x, u)+\bar{\rho}_{i} \bar{d}_{i}^{2}(v, y)\right] \geqq 0$ or $\rho_{i} \geqq 0$ and $\bar{\rho}_{i} \geqq 0, i=1,2, \ldots, k$,

(iv) $C_{x, u}(a)+a^{T} u \geqq 0, \forall a \in C_{1}^{*} \bar{C}_{v, y}(b)+b^{T} y \geqq 0, \forall b \in C_{2}^{*}$.

Then, $T-W \notin-K \backslash\{0\}$.

Proof: The proof follows on the lines of Theorem 3.1. 
Remark 3.1 Since every convex function is pseudoconvex, therefore the above weak duality theorem for the symmetric dual pair $(\mathrm{MFPP})_{T}$ and $(\mathrm{MFDP})_{W}$ can also be obtained under higher-order $K-(C, \alpha, \rho, d)$ pseudoconvexity assumptions.

Theorem 3.3 (Weak duality). Let $(x, y, T, z, r, \lambda, p)$ be feasible for (MFPP) $)_{T}$ and let $(u, v, W, w, t, \lambda, q)$ be feasible for (MFDP) $W$. Let $f(., v)+(.)^{T} w$ be higher order $K-(C, \alpha, \rho, d)-$ pseudoconvex at $u$ with respect to $\Phi(u, v, q),-W\left(g(., v)-(.)^{T} t\right)$ be higher-order $K-(C, \alpha, \rho, d)$ - pseudoconvex at $u$ with respect to $-W \Psi(u, v, q),-\left(f(x,)-.(.)^{T} z\right)$ be higher -order $K-(\bar{C}, \bar{\alpha}, \bar{\rho}, \bar{d})$-pseudoconvex at $y$ with respect to $-H(x, y, p)$ and $T\left(g(x,)+.(.)^{T} r\right)$ be higher -order $K-(\bar{C}, \bar{\alpha}, \bar{\rho}, \bar{d})$-pseudoconvex at $y$ with respect to $T G(x, y, p)$ where $C: R^{n} \times R^{n} \times R^{n} \rightarrow R$ and $\bar{C}: R^{m} \times R^{m} \times R^{m} \rightarrow R$. If the following conditions hold:

$$
\begin{gathered}
\text { either } \sum_{i=1}^{k} \lambda_{i}\left[\rho_{i} d_{i}^{2}(x, u)+\bar{\rho}_{i} \bar{d}_{i}^{2}(v, y)\right] \geqq 0 \quad \text { or } \rho_{i} \geqq 0 \text { and } \bar{\rho}_{i} \geqq 0, i=1,2, \ldots, k, \\
C_{x, u}(a)+a^{T} u \geqq 0, \forall a \in C_{1}^{*}, \bar{C}_{v, y}(b)+b^{T} y \geqq 0, \forall b \in C_{2}^{*} .
\end{gathered}
$$

Then, $T-W \notin-K \backslash\{0\}$.

Proof: The proof follows on the lines of Theorem 3.1.

Theorem 3.4 (Weak duality). Let $(x, y, T, z, r, \lambda, p)$ and $(u, v, W, w, t, \lambda, q)$ be feasible solutions of (MFPP) $)_{T}$ and $(\mathrm{MFDP})_{W}$, respectively. Suppose that

(i) $\left(f(., v)+(.)^{T} w\right)-W\left(g(., v)-(.)^{T} t\right)$ is higher-order $K-(C, \alpha, \rho, d)$ - pseudoconvex at $u$ with respect to $(\Phi(u, v, q)-W \Psi(u, v, q))$

(ii) $\left(-f(x,)+.(.)^{T} z\right)+T\left(g(x,)+.(.)^{T} r\right)$ is higher-order $K-(\bar{C}, \bar{\alpha}, \bar{\rho}, \bar{d})$-pseudoconvex at $y$ with respect to $-H(x, y, p)+T G(x, y, p)$,

(iii) either $\sum_{i=1}^{k} \lambda_{i}\left[\rho_{i} d_{i}^{2}(x, u)+\bar{\rho}_{i} \bar{d}_{i}^{2}(v, y)\right] \geqq 0$ or $\rho_{i} \geqq 0$ and $\bar{\rho}_{i} \geqq 0, i=1,2, \ldots, k$,

(iv) $C_{x, u}(a)+a^{T} u \geqq 0, \forall a \in C_{1}^{*} \bar{C}_{v, y}(b)+b^{T} y \geqq 0, \forall b \in C_{2}^{*}$.

Then, $T-W \notin-K \backslash\{0\}$.

Proof: The proof follows on the lines of Theorem 3.1.

Theorem 3.5 (Strong duality). Let $(\bar{x}, \bar{y}, \bar{T}, \bar{z}, \bar{r}, \bar{\lambda}, \bar{p})$ be an efficient solution of $(\mathrm{MFPP})_{T}$, and fix $\lambda=\bar{\lambda}$ in $(\mathrm{MFDP})_{W}$. If the following conditions hold

(i) $\nabla_{x} H_{i}(\bar{x}, \bar{y}, 0)=\nabla_{x} G_{i}(\bar{x}, \bar{y}, 0)=0, \nabla_{q_{i}} \Phi_{i}(\bar{x}, \bar{y}, 0)=\nabla_{q_{i}} \Psi_{i}(\bar{x}, \bar{y}, 0)=0, H_{i}(\bar{x}, \bar{y}, 0)=G_{i}(\bar{x}, \bar{y}, 0)=0$,

$\Phi_{i}(\bar{x}, \bar{y}, 0)=\Psi_{i}(\bar{x}, \bar{y}, 0)=0, \nabla_{y} H_{i}(\bar{x}, \bar{y}, 0)=\nabla_{y} G_{i}(\bar{x}, \bar{y}, 0)=0, \nabla_{p_{i}} H_{i}(\bar{x}, \bar{y}, 0)=\nabla_{p_{i}} G_{i}(\bar{x}, \bar{y}, 0)=$ $0, i=1,2, \ldots, k$,

(ii) for all $i \in\{1,2, \ldots, k\}$, the Hessian matrix $\nabla_{p_{i} p_{i}} H_{i}\left(\bar{x}, \bar{y}, \bar{p}_{i}\right)-\bar{T}_{i} \nabla_{p_{i} p_{i}} G_{i}\left(\bar{x}, \bar{y}, \overline{p_{i}}\right)$ is positive or negative definite,

(iii) the set of vectors $\left\{\nabla_{y} f_{i}(\bar{x}, \bar{y})-\bar{z}_{i}+\nabla_{p_{i}} H_{i}\left(\bar{x}, \bar{y}, \bar{p}_{i}\right)-\bar{T}_{i}\left(\nabla_{y} g_{i}(\bar{x}, \bar{y})+\bar{r}_{i}+\nabla_{p_{i}} G_{i}\left(\bar{x}, \bar{y}, \bar{p}_{i}\right)\right)\right\}_{i=1}^{k}$ is linearly independent,

(iv) for $\bar{p}_{i} \in R^{n}, \bar{p}_{i} \neq 0(i=1,2, \ldots, k)$ implies that $\sum_{i=1}^{k} \bar{p}_{i}^{T}\left[\nabla_{y} f_{i}(\bar{x}, \bar{y})-\bar{z}_{i}+\nabla_{p_{i}} H_{i}\left(\bar{x}, \bar{y}, \bar{p}_{i}\right)-\bar{T}_{i}\left(\nabla_{y} g_{i}(\bar{x}, \bar{y})+\bar{r}_{i}+\nabla_{p_{i}} G_{i}\left(\bar{x}, \bar{y}, \bar{p}_{i}\right)\right)\right] \neq 0$, 
(v) $\bar{T}_{i}>0, \forall i \in\{1,2, \ldots, k\}$

Then

(a) $\bar{p}_{i}=0, i=1,2, \ldots, k$,

(b) there exists $\bar{w}_{i} \in Q_{i}$ and $\bar{t}_{i} \in E_{i}, i=1,2, \ldots, k$ such that $(\bar{x}, \bar{y}, \bar{T}, \bar{w}, \bar{t}, \bar{\lambda}, \bar{q}=0)$ is a feasible solution of $(M F D P)_{W}$.

Furthermore, if the hypotheses in Theorems $(3.1)-(3.4)$ are satisfied, then $(\bar{x}, \bar{y}, \bar{T}, \bar{w}, \bar{t}, \bar{\lambda}, \bar{q}=0)$ is an efficient solution of $(M F D P)_{W}$, and the two objective values are equal.

Proof

Since $(\bar{x}, \bar{y}, \bar{T}, \bar{z}, \bar{r}, \bar{\lambda}, \bar{p})$ is an efficient solution of $(\mathrm{MFPP})_{T}$. Hence, by the Fritz John necessary optimality conditions [7], there exists $\alpha \in R^{k}, \beta \in R^{k}, \gamma \in C_{2}, \delta \in R, \xi \in R^{k}, \eta \in R, \bar{w}_{i} \in R^{n}$ and $\bar{t}_{i} \in R^{n}, i=1,2, \ldots, k$ such that

$$
\begin{aligned}
& (x-\bar{x})^{T}\left[\sum_{i=1}^{k} \beta_{i}\left(\nabla_{x} f_{i}(\bar{x}, \bar{y})+\bar{w}_{i}+\nabla_{x} H_{i}\left(\bar{x}, \bar{y}, \bar{p}_{i}\right)-\bar{T}_{i}\left(\nabla_{x} g_{i}(\bar{x}, \bar{y})-\bar{t}_{i}+\nabla_{x} G_{i}\left(\bar{x}, \bar{y}, \bar{p}_{i}\right)\right)\right)\right. \\
& +(\gamma-\delta \bar{y})^{T} \sum_{i=1}^{k} \bar{\lambda}_{i}\left(\nabla_{y x} f_{i}(\bar{x}, \bar{y}) \bar{T}_{i} \nabla_{y x} g_{i}(\bar{x}, \bar{y})\right) \\
& \quad+\sum_{i=1}^{k}\left(\nabla_{p_{i} x} H_{i}\left(\bar{x}, \bar{y}, \bar{p}_{i}\right)-\bar{T}_{i}\left(\nabla_{p_{i} x} G_{i}\left(\bar{x}, \bar{y}, \bar{p}_{i}\right)\right)^{T}\left((\gamma-\delta \bar{y}) \bar{\lambda}_{i}-\beta_{i} \bar{p}_{i}\right)\right] \geqq 0, \forall x \in C_{1},
\end{aligned}
$$

$$
\begin{gathered}
\sum_{i=1}^{k} \beta_{i}\left(\nabla_{y} f_{i}(\bar{x}, \bar{y})-\bar{z}_{i}+\nabla_{y} H_{i}\left(\bar{x}, \bar{y}, \bar{p}_{i}\right)-\bar{T}_{i}\left(\nabla_{y} g_{i}(\bar{x}, \bar{y})+\bar{r}_{i}+\nabla_{y} G_{i}\left(\bar{x}, \bar{y}, \bar{p}_{i}\right)\right)+\sum_{i=1}^{k} \bar{\lambda}_{i}\left(\nabla_{y y} f_{i}(\bar{x}, \bar{y})\right.\right. \\
\left.-\bar{T}_{i} \nabla_{y y} g_{i}(\bar{x}, \bar{y})\right)^{T}(\gamma-\delta \bar{y})+\sum_{i=1}^{k}\left(\nabla_{p_{i} y} H_{i}\left(\bar{x}, \bar{y}, \bar{p}_{i}\right)-\bar{T}_{i} \nabla_{p_{i} y} G_{i}\left(\bar{x}, \bar{y}, \bar{p}_{i}\right)\right)^{T}\left(-\beta_{i} \bar{p}_{i}+(\gamma-\delta \bar{y}) \bar{\lambda}_{i}\right) \\
-\delta \sum_{i=1}^{k} \bar{\lambda}_{i}\left[\nabla_{y} f_{i}(\bar{x}, \bar{y})-\bar{z}_{i}+\nabla_{p_{i}} H_{i}\left(\bar{x}, \bar{y}, \bar{p}_{i}\right)-\bar{T}_{i}\left(\nabla_{y} g_{i}(\bar{x}, \bar{y})+\bar{r}_{i}+\nabla_{p_{i}} G_{i}\left(\bar{x}, \bar{y}, \bar{p}_{i}\right)\right)\right]=0
\end{gathered}
$$

$$
\alpha_{i}-\beta_{i}\left(g_{i}(\bar{x}, \bar{y})-s\left(\bar{x} \mid E_{i}\right)+\bar{y}^{T} \bar{r}_{i}+G_{i}\left(\bar{x}, \bar{y}, \bar{p}_{i}\right)-\bar{p}_{i}{ }^{T} \nabla_{p_{i}} G_{i}\left(\bar{x}, \bar{y}, \bar{p}_{i}\right)\right)
$$

$$
-(\gamma-\delta \bar{y})^{T} \nabla_{y}\left(\bar{\lambda}_{i}\left(g_{i}(\bar{x}, \bar{y})+\bar{r}_{i}+\nabla_{p_{i}} G_{i}\left(\bar{x}, \bar{y}, \bar{p}_{i}\right)\right)=0, i=1,2, \ldots, k,\right.
$$

$$
(\gamma-\delta \bar{y})^{T}\left(\nabla_{y} f_{i}(\bar{x}, \bar{y})-\bar{z}_{i}+\nabla_{p_{i}} H_{i}\left(\bar{x}, \bar{y}, \bar{p}_{i}\right)\right.
$$

$$
-\bar{T}_{i}\left(\nabla_{y} g_{i}(\bar{x}, \bar{y})+\bar{r}_{i}+\nabla_{p_{i}} G_{i}\left(\bar{x}, \bar{y}, \bar{p}_{i}\right)-\xi_{i}+\eta\right)(\lambda-\bar{\lambda}) \geqq 0, \forall \lambda \in \operatorname{int} K^{*}, i=1,2, \ldots, k,
$$

$$
\left(\bar{\lambda}_{i}(\gamma-\delta \bar{y})-\beta_{i} \bar{p}_{i}\right)^{T}\left(\nabla_{p_{i} p_{i}} H_{i}\left(\bar{x}, \bar{y}, \bar{p}_{i}\right)-\bar{T}_{i} \nabla_{p_{i} p_{i}} G_{i}\left(\bar{x}, \bar{y}, \bar{p}_{i}\right)\right)=0, \quad i=1,2, \ldots, k,
$$

$$
\beta_{i} \bar{y}+(\gamma-\delta \bar{y}) \bar{\lambda}_{i} \in N_{D_{i}}\left(\bar{z}_{i}\right), i=1,2, \ldots, k
$$

$$
\beta_{i} \bar{T}_{i} \bar{y}+\bar{\lambda}_{i} \bar{T}_{i}(\gamma-\delta \bar{y}) \in N_{F_{i}}\left(\bar{r}_{i}\right), i=1,2, \ldots, k,
$$

$$
\gamma^{T} \sum_{i=1}^{k} \bar{\lambda}_{i}\left(\left(\nabla_{y} f_{i}(\bar{x}, \bar{y})-\bar{z}_{i}+\nabla_{p_{i}} H_{i}\left(\bar{x}, \bar{y}, \bar{p}_{i}\right)\right)-\bar{T}_{i}\left(\nabla_{y} g_{i}(\bar{x}, \bar{y})+\bar{r}_{i}+\nabla_{p_{i}} G_{i}\left(\bar{x}, \bar{y}, \bar{p}_{i}\right)\right)\right)=0
$$




$$
\begin{gathered}
\delta \bar{y}^{T} \sum_{i=1}^{k} \bar{\lambda}_{i}\left(\nabla_{y} f_{i}(\bar{x}, \bar{y})-\bar{z}_{i}+\nabla_{p_{i}} H_{i}\left(\bar{x}, \bar{y}, \bar{p}_{i}\right)-\bar{T}_{i}\left(\nabla_{y} g_{i}(\bar{x}, \bar{y})+\bar{r}_{i}+\nabla_{p_{i}} G_{i}\left(\bar{x}, \bar{y}, \overline{p_{i}}\right)\right)=0,\right. \\
\bar{\lambda}^{T} \xi=0, \\
\eta\left(\bar{\lambda}^{T} e-1\right)=0, \\
\bar{w}_{i} \in Q_{i}, \bar{t}_{i} \in E_{i}, \bar{x}^{T} t_{i}=s\left(\bar{x} \mid E_{i}\right), \bar{x}^{T} \bar{w}_{i}=s\left(\bar{x} \mid Q_{i}\right), i=1,2, \ldots, k, \\
(\alpha, \delta, \xi) \geqq 0, \quad(\alpha, \beta, \gamma, \delta, \xi, \eta) \neq 0 .
\end{gathered}
$$

Since $\bar{\lambda}>0$, and $\xi \geqq 0,(27)$ implies that $\xi=0$.

Equation (19) can be re-written as

$$
\begin{aligned}
& \sum_{i=1}^{k}\left(\beta_{i}-\delta \bar{\lambda}_{i}\right)\left(\left(\nabla_{y} f_{i}(\bar{x}, \bar{y})-\bar{z}_{i}+\nabla_{p_{i}} H_{i}\left(\bar{x}, \bar{y}, \bar{p}_{i}\right)\right)-\bar{T}_{i}\left(\nabla_{y} g_{i}(\bar{x}, \bar{y})+\bar{r}_{i}+\nabla_{p_{i}} G_{i}\left(\bar{x}, \bar{y}, \bar{p}_{i}\right)\right)\right) \\
& +\sum_{i=1}^{k} \beta_{i}\left(\left(\nabla_{y} H_{i}\left(\bar{x}, \bar{y}, \bar{p}_{i}\right)-\bar{T}_{i} \nabla_{y} G_{i}\left(\bar{x}, \bar{y}, \bar{p}_{i}\right)\right)-\left(\nabla_{p_{i}} H_{i}\left(\bar{x}, \bar{y}, \bar{p}_{i}\right)-\bar{T}_{i} \nabla_{p_{i}} G_{i}\left(\bar{x}, \bar{y}, \bar{p}_{i}\right)\right)\right. \\
& +\sum_{i=1}^{k} \bar{\lambda}_{i}\left(\left(\nabla_{y y} f_{i}(\bar{x}, \bar{y})-\bar{T}_{i} \nabla_{y y} g_{i}(\bar{x}, \bar{y})\right)^{T}(\gamma-\delta \bar{y})\right. \\
& \quad+\sum_{i=1}^{k}\left(\left(\nabla_{p_{i} y} H i\left(\bar{x}, \bar{y}, \bar{p}_{i}\right)-\bar{T}_{i} \nabla_{p_{i} y} G_{i}\left(\bar{x}, \bar{y}, \bar{p}_{i}\right)\right)^{T}\left(-\beta_{i} \bar{p}_{i}+(\gamma-\delta \bar{y}) \bar{\lambda}_{i}\right)=0 .\right.
\end{aligned}
$$

Inequality (21) is equivalent to

$$
\begin{aligned}
(\gamma-\delta \bar{y})^{T}\left(\nabla_{y} f_{i}(\bar{x}, \bar{y})\right. & -\bar{z}_{i}+\nabla_{p_{i}} H_{i}\left(\bar{x}, \bar{y}, \bar{p}_{i}\right) \\
& -\bar{T}_{i}\left(\nabla_{y} g_{i}(\bar{x}, \bar{y})+\bar{r}_{i}+\nabla_{p_{i}} G_{i}\left(\bar{x}, \bar{y}, \bar{p}_{i}\right)\right)-\xi_{i}+\eta=0, i=1,2, \ldots, k .
\end{aligned}
$$

By hypothesis (ii) and (22), we have

$$
\bar{\lambda}_{i}(\gamma-\delta \bar{y})=\beta_{i} \bar{p}_{i}, i=1,2, \ldots, k .
$$

Now, we claim that $\beta_{i} \neq 0, \forall i$. If possible, let $\beta_{t_{0}}=0$ for some $t_{0}, 1 \leq t_{0} \leq k$, then from $\bar{\lambda}_{t_{0}}>0$ and equation (33), we have

$$
\gamma=\delta \bar{y}
$$

Using (33) and (34), we obtain $\beta_{i} \bar{p}_{i}=0, i=1,2, \ldots, k$. Hence, by hypothesis $(i)$, we get

$$
\sum_{i=1}^{k} \beta_{i}\left(\left(\nabla_{y} H_{i}\left(\bar{x}, \bar{y}, \bar{p}_{i}\right)-\bar{T}_{i} \nabla_{y} G_{i}\left(\bar{x}, \bar{y}, \bar{p}_{i}\right)\right)-\left(\nabla_{p_{i}} H_{i}\left(\bar{x}, \bar{y}, \bar{p}_{i}\right)-\bar{T}_{i} \nabla_{p_{i}} G_{i}\left(\bar{x}, \bar{y}, \bar{p}_{i}\right)\right)\right)=0 .
$$

Using (33)-(35) in (31), we obtain

$$
\sum_{i=1}^{k}\left(\beta_{i}-\delta \bar{\lambda}_{i}\right)\left(\nabla_{y} f_{i}(\bar{x}, \bar{y})-\bar{z}_{i}+\nabla_{p_{i}} H_{i}\left(\bar{x}, \bar{y}, \bar{p}_{i}\right)-\bar{T}_{i}\left(\nabla_{y} g_{i}(\bar{x}, \bar{y})+\bar{r}_{i}+\nabla_{p_{i}} G_{i}\left(\bar{x}, \bar{y}, \bar{p}_{i}\right)\right)\right)=0
$$


which by hypothesis (iii), follows that

$$
\bar{\beta}_{i}-\delta \bar{\lambda}_{i}=0, i=1,2, \ldots, k .
$$

Now, for $i=t_{0}$, we have $\delta \bar{\lambda}_{i}=0$. This implies $\delta=0$. since $\bar{\lambda}>0$. Hence, from (37) $\beta_{i}=0, \forall i$. Thus, from relation (20), (34) and (37), we get $\alpha_{i}=0, i=1,2, \ldots, k$. Also, from relations (20) and (34), we get $\eta=0$ and $\gamma=0$, respectively, which contradicts the fact that $(\alpha, \beta, \gamma, \delta, \xi, \eta) \neq 0$. Hence $\beta_{i} \neq 0, i=1,2, \ldots, k$.

Now, equation (32) reduces to

$$
\begin{aligned}
(\gamma-\delta \bar{y})^{T}\left(\nabla_{y} f_{i}(\bar{x}, \bar{y})-\bar{z}_{i}+\nabla_{p_{i}} H_{i}\left(\bar{x}, \bar{y}, \bar{p}_{i}\right)\right. & \\
& -\bar{T}_{i}\left(\nabla_{y} g_{i}(\bar{x}, \bar{y})+\bar{r}_{i}+\nabla_{p_{i}} G_{i}\left(\bar{x}, \bar{y}, \bar{p}_{i}\right)\right)+\eta=0, i=1,2, \ldots, k .
\end{aligned}
$$

Multiplying by $\bar{\lambda}_{i}$ and summing over $i$, we get

$$
\begin{aligned}
(\gamma-\delta \bar{y})^{T} \sum_{i}^{k} \bar{\lambda}_{i}\left(\nabla_{y} f_{i}(\bar{x}, \bar{y})\right. & -\bar{z}_{i}+\nabla_{p_{i}} H_{i}\left(\bar{x}, \bar{y}, \bar{p}_{i}\right) \\
& -\bar{T}_{i}\left(\nabla_{y} g_{i}(\bar{x}, \bar{y})+\bar{r}_{i}+\nabla_{p_{i}} G_{i}\left(\bar{x}, \bar{y}, \bar{p}_{i}\right)\right)+\eta \bar{\lambda}^{T} e_{k}=0 .
\end{aligned}
$$

Subtracting (26) from (25), we get

$$
(\gamma-\delta \bar{y})^{T} \sum_{i}^{k} \bar{\lambda}_{i}\left(\nabla_{y} f_{i}(\bar{x}, \bar{y})-\bar{z}_{i}+\nabla_{p_{i}} H_{i}\left(\bar{x}, \bar{y}, \bar{p}_{i}\right)-\bar{T}_{i}\left(\nabla_{y} g_{i}(\bar{x}, \bar{y})+\bar{r}_{i}+\nabla_{p_{i}} G_{i}\left(\bar{x}, \bar{y}, \bar{p}_{i}\right)\right)=0 .\right.
$$

Using (40) in (39), we get, $\eta=0$.

Now, equation, yield

$$
(\gamma-\delta \bar{y})^{T}\left(\nabla_{y} f_{i}(\bar{x}, \bar{y})-\bar{z}_{i}+\nabla_{p_{i}} H_{i}\left(\bar{x}, \bar{y}, \bar{p}_{i}\right)-\bar{T}_{i}\left(\nabla_{y} g_{i}(\bar{x}, \bar{y})+\bar{r}_{i}+\nabla_{p_{i}} G_{i}\left(\bar{x}, \bar{y}, \bar{p}_{i}\right)\right)=0, i=1,2, \ldots, k .\right.
$$

Since $\bar{\lambda}>0$, using (33) in (34), we get

$$
\beta_{i} \bar{p}_{i}^{T}\left[\nabla_{y} f_{i}(\bar{x}, \bar{y})-\bar{z}_{i}+\nabla_{p_{i}} H_{i}\left(\bar{x}, \bar{y}, \bar{p}_{i}\right)-\bar{T}_{i}\left(\nabla_{y} g_{i}(\bar{x}, \bar{y})+\bar{r}_{i}+\nabla_{p_{i}} G_{i}\left(\bar{x}, \bar{y}, \bar{p}_{i}\right)\right)\right]=0, i=1,2, \ldots, k .
$$

Since $\beta_{i} \neq 0, i=1,2, \ldots, k$, we obtain

$$
\bar{p}_{i}^{T}\left[\nabla_{y} f_{i}(\bar{x}, \bar{y})-\bar{z}_{i}+\nabla_{p_{i}} H_{i}\left(\bar{x}, \bar{y}, \bar{p}_{i}\right)-\bar{T}_{i}\left(\nabla_{y} g_{i}(\bar{x}, \bar{y})+\bar{r}_{i}+\nabla_{p_{i}} G_{i}\left(\bar{x}, \bar{y}, \bar{p}_{i}\right)\right)\right]=0, i=1,2, \ldots, k,
$$

or

$$
\sum_{i=1}^{k} \bar{p}_{i}^{T}\left[\nabla_{y} f_{i}(\bar{x}, \bar{y})-\bar{z}_{i}+\nabla_{p_{i}} H_{i}\left(\bar{x}, \bar{y}, \bar{p}_{i}\right)-\bar{T}_{i}\left(\nabla_{y} g_{i}(\bar{x}, \bar{y})+\bar{r}_{i}+\nabla_{p_{i}} G_{i}\left(\bar{x}, \bar{y}, \bar{p}_{i}\right)\right)\right]=0 .
$$

By the hypothesis $(i v)$, we have $\bar{p}_{i}=0, i=1,2, \ldots, k$. Further using, hypothesis $(i)$, using (32)-(33) in (18)-(19), we get

$$
\begin{gathered}
(x-\bar{x})^{T}\left[\sum_{i=1}^{k} \beta_{i}\left(\nabla_{x} f_{i}(\bar{x}, \bar{y})+\bar{w}_{i}-\bar{T}_{i}\left(\nabla_{x} g_{i}(\bar{x}, \bar{y})-\bar{t}_{i}\right)\right)\right] \geqq 0, \forall x \in C_{1} . \\
\sum_{i=1}^{k}\left(\beta_{i}-\delta \bar{\lambda}_{i}\right)\left[\nabla_{y} f_{i}(\bar{x}, \bar{y})-\bar{z}_{i}-\bar{T}_{i}\left(\nabla_{y} g_{i}(\bar{x}, \bar{y})+\bar{r}_{i}\right)\right]=0 .
\end{gathered}
$$


Using hypothesis (iii) in (46), we have

$$
\beta_{i}=\delta \bar{\lambda}_{i}, i=1,2, \ldots, k
$$

Since $\beta_{i} \neq 0, \bar{\lambda}_{i}>0, i=1,2, \ldots, k$ and $\delta \geqq 0$, this implies that $\beta_{i}>0, \forall i$. Now, using (47) in (46), we obtain

$$
(x-\bar{x})^{T}\left[\sum_{i=1}^{k} \bar{\lambda}_{i}\left(\nabla_{x} f_{i}(\bar{x}, \bar{y})+\bar{w}_{i}-\bar{T}_{i}\left(\nabla_{x} g_{i}(\bar{x}, \bar{y})-\bar{t}_{i}\right)\right)\right] \geqq 0, \forall x \in C_{1} .
$$

Let $x \in C_{1}$. Then $x+\bar{x} \in C_{1}$, as $C_{1}$ is a closed convex cone. On substituting $x+\bar{x}$ in place of $x$ in (48), we have

$$
x^{T} \sum_{i=1}^{k} \bar{\lambda}_{i}\left[\left(\nabla_{x} f_{i}(\bar{x}, \bar{y})+\bar{w}_{i}\right)-\bar{T}_{i}\left(\nabla_{x} g_{i}(\bar{x}, \bar{y})-\bar{t}_{i}\right)\right] \geqq 0,
$$

which in turn implies that for all $x \in C_{1}$, we have

$$
\left.\sum_{i=1}^{k} \bar{\lambda}_{i}\left[\left(\nabla_{x} f_{i}(\bar{x}, \bar{y})+\bar{w}_{i}\right)-\bar{T}_{i}\left(\nabla_{x} g_{i}(\bar{x}, \bar{y})-\bar{t}_{i}\right)\right)\right] \in C_{1}^{*} .
$$

Also by letting $x=0$ and $x=2 \bar{x}$, simultaneously in (48), yields

$$
\left.\bar{x}^{T} \sum_{i=1}^{k} \bar{\lambda}_{i}\left(\nabla_{x} f_{i}(\bar{x}, \bar{y})+\bar{w}_{i}\right)-\bar{T}_{i}\left(\nabla_{x} g_{i}(\bar{x}, \bar{y})-\bar{t}_{i}\right)\right)=0 .
$$

Using $\bar{p}_{i}=0$ in (33), we get, $\gamma=\delta \bar{y}$ and $\delta>0$, we have

$$
\bar{y}=\frac{\gamma}{\delta} \in C_{2} .
$$

Since $\beta>0$ by (23) and the fact that $\gamma=\delta \bar{y}$, we get $\bar{y} \in N_{D_{i}}\left(\bar{z}_{i}\right), i=1,2, \ldots, k$. This implies

$$
\bar{y}^{T} \bar{z}_{i}=s\left(\bar{y} \mid D_{i}\right), i=1,2, \ldots, k .
$$

By (24) and hypothesis $(v)$, we have $\bar{y} \in N_{F_{i}}\left(\bar{r}_{i}\right), i=1,2, \ldots ., k$. Hence,

$$
\bar{y}^{T} \bar{r}_{i}=s\left(\bar{y} \mid F_{i}\right), i=1,2, \ldots . ., k .
$$

Combining (29),(52)-(53) and given equation (1), reduce to

$$
\left(f_{i}(\bar{x}, \bar{y})+\bar{x}^{T} \bar{w}_{i}-s\left(\bar{y} \mid D_{i}\right)\right)-\bar{T}_{i}\left(g_{i}(\bar{x}, \bar{y})-\bar{x}^{T} \bar{t}_{i}-s\left(\bar{y} \mid F_{i}\right)\right)=0, i=1,2, \ldots ., k .
$$

Combining this with (52)-(54), shows that $(\bar{x}, \bar{y}, \bar{T}, \bar{w}, \bar{t}, \bar{\lambda}, \bar{q}=0)$ is a feasible solution of $(M F D P)_{V}$.

Under the assumptions Theorems $(3.1)-(3.4)$, if $(\bar{x}, \bar{y}, \bar{T}, \bar{w}, \bar{t}, \bar{\lambda}, \bar{q}=0)$ is not an efficient solution of $(M F D P)_{W}$, then there exists other feasible solution $(u, v, W, \bar{w}, \bar{t}, \bar{\lambda}, q)$, of $(M F D P)_{W}$, such that $W-\bar{T} \in K \backslash\{0\}$.

Since $(\bar{x}, \bar{y}, \bar{T}, \bar{w}, \bar{t}, \bar{\lambda}, \bar{p})$ is a feasible solution of $(M F P P)_{T}$, by Weak duality theorem, we have $W-\bar{T} \notin K \backslash\{0\}$, hence the contradiction implies that $(\bar{x}, \bar{y}, \bar{T}, \bar{w}, \bar{t}, \bar{\lambda}, \bar{q}=0)$ is an efficient solution of $(M F D P)_{W}$. Hence, the result.

Theorem 3.6 (Strong duality). Let $(\bar{x}, \bar{y}, \bar{T}, \bar{z}, \bar{r}, \bar{\lambda}, \bar{p})$ be efficient solution of $(\mathrm{MFPP})_{T}$, and fix $\lambda=\bar{\lambda}$ in $(\mathrm{MFDP})_{W}$. Suppose that 
(i) $\nabla_{x} H_{i}(\bar{x}, \bar{y}, 0)=\nabla_{x} G_{i}(\bar{x}, \bar{y}, 0)=0, \nabla_{q_{i}} \Phi_{i}(\bar{x}, \bar{y}, 0)=\nabla_{q_{i}} \Psi_{i}(\bar{x}, \bar{y}, 0)=0, H_{i}(\bar{x}, \bar{y}, 0)=G_{i}(\bar{x}, \bar{y}, 0)=$ $0, \Phi_{i}(\bar{x}, \bar{y}, 0)=\Psi_{i}(\bar{x}, \bar{y}, 0)=0, \nabla_{y} H_{i}(\bar{x}, \bar{y}, 0)=\nabla_{y} G_{i}(\bar{x}, \bar{y}, 0)=0, \nabla_{p_{i}} H_{i}(\bar{x}, \bar{y}, 0)=\nabla_{p_{i}} G_{i}(\bar{x}, \bar{y}, 0)=$ $0, i=1,2, \ldots, k$.

(ii) $\bar{T}_{i}>0, \forall i \in 1,2, \ldots \ldots, k$,

(iii) $\nabla_{p_{i} p_{i}} H_{i}\left(\bar{x}, \bar{y}, \bar{p}_{i}\right)-\bar{T}_{i} \nabla_{p_{i} p_{i}} G_{i}\left(\bar{x}, \bar{y}, \overline{p_{i}}\right)$ is nonsingular $\forall i=1,2, \ldots ., k$,

(iv) $\sum_{i=1}^{k} \bar{\lambda}_{i}\left(\nabla_{y y} f_{i}(\bar{x}, \bar{y})-\bar{T}_{i} \nabla_{y y} g_{i}(\bar{x}, \bar{y})\right)$ is positive definite and $\bar{p}_{i}^{T}\left(\left(\nabla_{y} H_{i}\left(\bar{x}, \bar{y}, \bar{p}_{i}\right)-\bar{T}_{i} \nabla_{y} G_{i}\left(\bar{x}, \bar{y}, \bar{p}_{i}\right)\right)-\right.$ $\left.\left(\nabla_{p_{i}} H_{i}\left(\bar{x}, \bar{y}, \bar{p}_{i}\right)-\bar{T}_{i} \nabla_{p_{i}} G_{i}\left(\bar{x}, \bar{y}, \bar{p}_{i}\right)\right)\right) \geqq 0, \quad \forall i=1,2, \ldots ., k$, or $\sum_{i=1}^{k} \bar{\lambda}_{i}\left(\left(\nabla_{y y} f_{i}(\bar{x}, \bar{y})-\bar{T}_{i} \nabla_{y y} g_{i}(\bar{x}, \bar{y})\right)\right.$ is negative definite and

$\bar{p}_{i}^{T}\left(\nabla_{y} H_{i}\left(\bar{x}, \bar{y}, \bar{p}_{i}\right)-\bar{T}_{i} \nabla_{y} G_{i}\left(\bar{x}, \bar{y}, \bar{p}_{i}\right)\right)-\left(\nabla_{p_{i}} H_{i}\left(\bar{x}, \bar{y}, \bar{p}_{i}\right)-\bar{T}_{i} \nabla_{p_{i}} G_{i}\left(\bar{x}, \bar{y}, \overline{p_{i}}\right)\right) \leqq 0, \forall i=1,2, \ldots ., k$.

$(v)$ the set of vectors $\left\{\nabla_{y} f_{i}(\bar{x}, \bar{y})-\bar{z}_{i}+\nabla_{p_{i}} H_{i}\left(\bar{x}, \bar{y}, \bar{p}_{i}\right)-\bar{T}_{i}\left(\nabla_{y} g_{i}(\bar{x}, \bar{y})+\bar{r}_{i}+\nabla_{p_{i}} G_{i}\left(\bar{x}, \bar{y}, \overline{p_{i}}\right): i=\right.\right.$ $1,2, \ldots \ldots, k\}$ is linearly independent.

Then $\bar{p}=0$, and there exists $\bar{w}_{i} \in Q_{i}$ and $\bar{t}_{i} \in E_{i}, i=1,2, \ldots \ldots, k$ such that $(\bar{x}, \bar{y}, \bar{T}, \bar{w}, \bar{t}, \bar{\lambda}, \bar{q}=0)$ is a feasible solution of $(M F D P)_{W}$. Furthermore, if the hypotheses in theorems (3.1) - (3.4) are satisfied, then $(\bar{x}, \bar{y}, \bar{T}, \bar{w}, \bar{t}, \bar{\lambda}, \bar{q}=0)$ is efficient solution of $(M F D P)_{W}$, and the two objective values are equal.

Proof: The proof follows Theorem 3.5.

Theorem 3.7 (Converse duality). Let $(\bar{u}, \bar{v}, \bar{W}, \bar{w}, \bar{t}, \bar{\lambda}, \bar{q})$ be efficient solution of $(\mathrm{MFPP})_{W}$, and fix $\lambda=\bar{\lambda}$ in $(\mathrm{MFDP})_{T}$. If the following conditions hold

(i) $\nabla_{x} \Phi_{i}(\bar{u}, \bar{v}, 0)=\nabla_{x} \Psi_{i}(\bar{u}, \bar{v}, 0)=0, \nabla_{q_{i}} \Phi_{i}(\bar{u}, \bar{v}, 0)=\nabla_{q_{i}} \Psi_{i}(\bar{u}, \bar{v}, 0)=0, \quad H_{i}(\bar{u}, \bar{v}, 0)=G_{i}(\bar{u}, \bar{v}, 0)=$ $0, \Phi_{i}(\bar{u}, \bar{v}, 0)=\Psi_{i}(\bar{u}, \bar{v}, 0)=0, \nabla_{y} \Phi_{i}(\bar{u}, \bar{v}, 0) \quad=\nabla_{y} \Psi_{i}(\bar{u}, \bar{v}, 0)=0, \nabla_{p_{i}} H_{i}(\bar{u}, \bar{v}, 0)=\nabla_{p_{i}} G_{i}(\bar{u}, \bar{v}, 0)=$ $0, i=1,2, \ldots, k$.

(ii) for all $i \in\{1,2, \ldots, k\}$, the Hessian matrix $\nabla_{p_{i} p_{i}} \Phi_{i}\left(\bar{u}, \bar{v}, \overline{q_{i}}\right)-\bar{W}_{i} \nabla_{p_{i} p_{i}} \Psi_{i}\left(\bar{u}, \bar{v}, \overline{q_{i}}\right)$ is positive or negative definite;

(iii) the set of vectors $\left.\left\{\nabla_{x} f_{i}(\bar{u}, \bar{v})+\bar{w}_{i}+\nabla_{q_{i}} \Phi_{i}\left(\bar{u}, \bar{v}, \bar{q}_{i}\right)-\bar{W}_{i}\left(\nabla_{x} g_{i}(\bar{u}, \bar{v})-\bar{t}_{i}+\nabla_{q_{i}} \Psi_{i}\left(\bar{u}, \bar{v}, \bar{q}_{i}\right)\right)\right)\right\}_{i=1}^{k}$ is linearly independent;

$(i v)$ for $\quad \bar{q}_{i} \in R^{n}, \quad \bar{q}_{i} \neq 0, \quad(i=1,2, \ldots, k) \quad$ implies $\quad$ that $\sum_{i=1}^{k} \bar{q}_{i}^{T}\left[\nabla_{x} f_{i}(\bar{u}, \bar{v})+\bar{w}_{i}+\nabla_{q_{i}} \Phi_{i}\left(\bar{u}, \bar{v}, \bar{q}_{i}-\right.\right.$ $\left.\bar{W}_{i}\left(\nabla_{x} g_{i}(\bar{u}, \bar{v})-\bar{t}_{i}+\nabla_{q_{i}} \Psi_{i}\left(\bar{u}, \bar{v}, \bar{q}_{i}\right)\right)\right] \neq 0$,

(v) $\bar{W}_{i}>0, \forall i \in 1,2, \ldots, k$.

Then

(a) $\bar{q}_{i}=0, i=1,2, \ldots, k$;

(b) there exists $\bar{z}_{i} \in D_{i}$ and $\bar{r}_{i} \in F_{i}, i=1,2, \ldots, k$ such that $(\bar{u}, \bar{v}, \bar{V}, \bar{z}, \bar{r}, \bar{\lambda}, \bar{p}=0)$ is a feasible solution of $(M F D P)_{W}$.

Furthermore, if the hypotheses in Theorems $(3.1)-(3.4)$ are satisfied, then $(\bar{u}, \bar{v}, \bar{W}, \bar{z}, \bar{r}, \bar{\lambda}, \bar{p}=0)$ is an efficient solution of $(M F D P)_{T}$, and the two objective values are equal.

Proof: The proof follows Theorem 3.5.

Theorem 3.8 (Converse duality). Let $(\bar{u}, \bar{v}, \bar{V}, \bar{w}, \bar{t}, \bar{\lambda}, \bar{q})$ be efficient solution of $(\mathrm{MFPP})_{W}$, and fix $\lambda=\bar{\lambda}$ in $(\text { MFDP) })_{T}$. Suppose that

(i) $\nabla_{x} \Phi_{i}(\bar{u}, \bar{v}, 0)=\nabla_{x} \Psi_{i}(\bar{u}, \bar{v}, 0)=0, \nabla_{q_{i}} \Phi_{i}(\bar{u}, \bar{v}, 0)=\nabla_{q_{i}} \Psi_{i}(\bar{u}, \bar{v}, 0)=0, H_{i}(\bar{u}, \bar{v}, 0)=G_{i}(\bar{u}, \bar{v}, 0)=0$, 


$$
\begin{aligned}
& \Phi_{i}(\bar{u}, \bar{v}, 0)=\Psi_{i}(\bar{u}, \bar{v}, 0)=0, \nabla_{y} \Phi_{i}(\bar{u}, \bar{v}, 0) \quad=\nabla_{y} \Psi_{i}(\bar{u}, \bar{v}, 0)=0, \nabla_{p_{i}} H_{i}(\bar{u}, \bar{v}, 0)=\nabla_{p_{i}} G_{i}(\bar{u}, \bar{v}, 0)= \\
& 0, i=1,2, \ldots, k .
\end{aligned}
$$

(ii) $\bar{W}_{i}>0, \forall i \in 1,2, \ldots \ldots, k$,

(iii) $\nabla_{q_{i} q_{i}} \Phi_{i}\left(\bar{u}, \bar{v}, \bar{q}_{i}\right)-\bar{W}_{i} \nabla_{q_{i} q_{i}} \Psi_{i}\left(\bar{u}, \bar{v}, \bar{q}_{i}\right)$ is nonsingular $\forall i=1,2, \ldots . ., k$,

$(i v) \sum_{i=1}^{k} \bar{\lambda}_{i}\left(\nabla_{x x} f_{i}(\bar{u}, \bar{v})-\bar{W}_{i} \nabla_{x x} g_{i}(\bar{u}, \bar{v})\right)$ is positive definite and $\bar{q}_{i}^{T}\left(\left(\nabla_{x} \Phi_{i}\left(\bar{u}, \bar{v}, \bar{q}_{i}\right)-\bar{W}_{i} \nabla_{x} \Psi_{i}\left(\bar{u}, \bar{v}, \bar{q}_{i}\right)\right)\right.$ $-\left(\left(\nabla_{q_{i}} \Phi_{i}\left(\bar{u}, \bar{v}, \bar{q}_{i}\right)-\bar{W}_{i} \nabla_{q_{i}} \Psi_{i}\left(\bar{u}, \bar{v}, \bar{q}_{i}\right)\right) \geqq 0, \forall i=1,2, \ldots, k\right.$,

or $\sum_{i=1}^{k} \bar{\lambda}_{i}\left(\nabla_{x x} f_{i}(\bar{u}, \bar{v})-\bar{V} \nabla_{x x} g_{i}(\bar{u}, \bar{v})\right)$ is negative definite and $\bar{q}_{i}^{T}\left(\left(\nabla_{x} \Phi_{i}\left(\bar{u}, \bar{v}, \bar{q}_{i}\right)-\bar{W}_{i} \nabla_{x} \Psi_{i}\left(\bar{u}, \bar{v}, \overline{q_{i}}\right)\right)-\right.$ $\left(\left(\nabla_{q_{i}} \Phi_{i}\left(\bar{u}, \bar{v}, \bar{q}_{i}\right)-\bar{W}_{i} \nabla_{q_{i}} \Phi_{i} \Psi_{i}\left(\bar{u}, \bar{v}, \overline{q_{i}}\right)\right) \leqq 0, \forall i=1,2, \ldots . ., k\right.$.

$(v)$ the set of vectors $\left\{\nabla_{x} f_{i}(\bar{u}, \bar{v})+\bar{w}_{i}+\nabla_{q_{i}} \Phi_{i}\left(\bar{u}, \bar{v}, \bar{q}_{i}\right)-\bar{W}_{i}\left(\nabla_{x} g_{i}(\bar{x}, \bar{y})-\bar{t}_{i}+\nabla_{q_{i}} \Psi_{i}\left(\bar{u}, \bar{v}, \overline{q_{i}}\right)\right): i=\right.$ $1,2, \ldots, k\}$ is linearly independent.

Then $\bar{q}=0$ and there exists $\bar{z}_{i} \in D_{i}$ and $\bar{r}_{i} \in F_{i}, \quad i=1,2, \ldots, k$ such that $(\bar{u}, \bar{v}, \bar{W}, \bar{z}, \bar{r}, \bar{\lambda}, \bar{p}=0)$ is a feasible solution of $(\mathrm{MFDP})_{T}$. Furthermore, if the hypotheses in Theorems (3.1) - (3.4) are satisfied, then $(\bar{u}, \bar{v}, \bar{W}, \bar{z}, \bar{r}, \bar{\lambda}, \bar{p}=0)$ is an efficient solution of $(\mathrm{MFDP})_{T}$ and the two objective values are equal.

Proof: The proof follows Theorem 3.5.

\section{Conclusion}

In this article, we have considered a pair of Mond-Weir type nondifferentiable higher order fractional symmetric dual program with cone constraints and discussed duality theorems under higher order $K-$

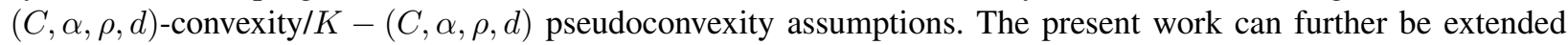
to nondifferentiable higher order symmetric fractional programming problem over cones under generalized assumptions. This will orient the future task of the authors.

\section{Acknowledgement}

The authors are thankful to the anonymous learned referees and editor for their valuable suggestions, which have substantially improved the presentation of the manuscript. The first author gratefully acknowledges the "J.C. Bose University of Science and Technology, YMCA, Faridabad-121 006, India”.

\section{REFERENCES}

1. O.L. Mangasarian, Second-and higher-order duality in nonlinear programming, Journal of Mathematical Analysis and Applications, vol. 51, no. 1, 607-620, 1975.

2. X.H. Chen, Higher-order symmetric duality in nondifferentiable multiobjective programming problems, Journal of Mathematical Analysis and Applications, vol. 290, no.12, pp. 423-435, 2004.

3. Mohamed Abd El-Hady Kassem, Higher-order symmetric duality in vector optimization problem involving generalized cone-invex functions, Appl. Math. Comput., vol. 2, no. 2, pp. 405-409, 2009.

4. S.K. Suneja, M.K. Srivastava and M. Bhatia, Duality in Multiobjective Fractional Programming with Support Functions, Journal of Mathematical Analysis and Application., vol. 347, no. 1, pp. 8-17, 2008.

5. G. Ying, Higher-order symmetric duality for a class of multiobjective fractional programming problems, Journal of Inequalities and Applications, 142, doi.org/10.1186/1029-242X-2012-142, 2012.

6. R. Dubey and S.K. Gupta, Duality for a nondifferentiable multiobjective higher-order symmetric fractional programming problem with cone constraints, Journal of Nonlinear Analysis and Optimization, Vol.7, no.1, 1-15, 2016.

7. B.D. Craven, Lagrange conditions and quasiduality, Bulletin of the Australian Mathematical Society, vol. 16, no.3, pp.325-339, 1977. 
8. S.K. Gupta and N. Kailey, Second-order multiobjective symmetric duality involving cone-bonvex functions, Journal of Global Optimization,vol. 55, no.1, pp.125-140, 2013.

9. S.K. Gupta, N. Kailey and M.K. Sharma, Multiobjective second-order nondifferentiable symmetric duality involving $(F, \alpha, \rho, d)$ convex function, Journal of applied mathematics and information sciences, vol.28, pp. 1395-1408, 2010.

10. X.J. Long, Optimality conditions and duality for nondifferentiable multiobjective fractional programming problems with $(C, \alpha, \rho, d)$ convexity, Journal of Optimization Theory and Applications, vol.148, no. 1, pp. 197-208, 2011.

11. R. Dubey, S.K. Gupta and M.A. Khan, Optimality and duality results for a nondifferentiable multiobjective fractional programming problem, Journal of Inequalities and Applications, vol.354, DOI 10.1186/s13660-015-0876-0, 2015.

12. R. Dubey and S.K. Gupta, Duality for a nondifferentiable multiobjective higher-order symmetric fractional programming problems with cone constraints, Journal of Nonlinear Analysis and Applications, vol.7, pp.1-15, pp. 294-311, DOI: 10.4236/ajor.2018.84017, 2016.

13. Vandana, R. Dubey, Deepmala, L.N. Mishra and V.N. Mishra, Duality relations for a class of a multiobjective fractional programming problem involving support functions, American Journal of Operations Research, vol. 8 , pp. 294-311, DOI: 10.4236/ajor.2018.84017, 2018.

14. R. Dubey, Vandana and V.N. Mishra, Second order multiobjective symmetric programming problem and duality relations under $\left(F, G_{f}\right)$-convexity, Global Journal of Engineering Science and Researches, vol. 5, no. 8, pp.187-199, DOI: 10.5281/zenodo.1341853, 2018.

15. R. Dubey, V.N. Mishra and P. Tomar, Duality relations for second-order programming problem under $(G, f)$-bonvexity assumptions, Asian-European Journal of Mathematics, vol.13, no. 1, pp.1-17, DOI: 10.1142/S1793557120500448, 2020. 